\title{
Electronic Structure Calculations in Arbitrary Electrostatic Environment
}

\section{Citation}

Watson, Mark A., Dmitrij Rappoport, Elizabeth M. Y. Lee, Roberto Olivares-Amaya, and Alán Aspuru-Guzik. 2012. Electronic Structure Calculations in Arbitrary Electrostatic Environment. Journal of Chemical Physics 136(2): 024101.

\section{Published Version}

doi:10.1063/1.3670417

\section{Permanent link}

http://nrs.harvard.edu/urn-3:HUL.InstRepos:8366165

\section{Terms of Use}

This article was downloaded from Harvard University's DASH repository, and is made available under the terms and conditions applicable to Open Access Policy Articles, as set forth at http:// nrs.harvard.edu/urn-3:HUL.InstRepos:dash.current.terms-of-use\#OAP

\section{Share Your Story}

The Harvard community has made this article openly available.

Please share how this access benefits you. Submit a story.

Accessibility 


\section{AIP $\begin{gathered}\text { the coumalot } \\ \text { chemical Physics }\end{gathered}$}

\section{Electronic structure calculations in arbitrary electrostatic environments}

Mark A. Watson, Dmitrij Rappoport, Elizabeth M. Y. Lee, Roberto Olivares-Amaya, and Alán Aspuru-Guzik

Citation: J. Chem. Phys. 136, 024101 (2012); doi: 10.1063/1.3670417

View online: http://dx.doi.org/10.1063/1.3670417

View Table of Contents: http://jcp.aip.org/resource/1/JCPSA6/v136/i2

Published by the American Institute of Physics.

\section{Additional information on J. Chem. Phys.}

Journal Homepage: http://jcp.aip.org/

Journal Information: http://jcp.aip.org/about/about_the_journal

Top downloads: http://jcp.aip.org/features/most_downloaded

Information for Authors: http://jcp.aip.org/authors

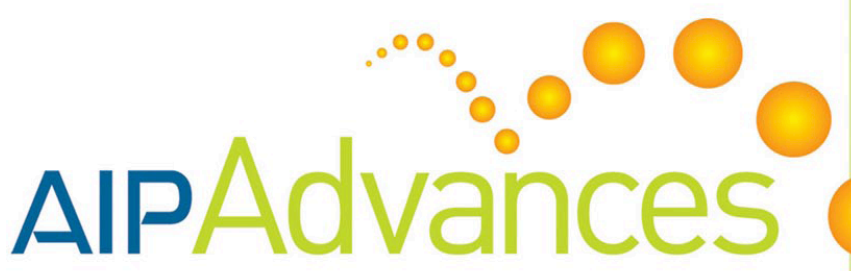

Explore AIP's new open-access journal

Article-level metrics now available

Join the conversation!

Submit Now 


\title{
Electronic structure calculations in arbitrary electrostatic environments
}

\author{
Mark A. Watson, Dmitrij Rappoport, Elizabeth M. Y. Lee, ${ }^{\text {a) }}$ Roberto Olivares-Amaya, \\ and Alán Aspuru-Guzik ${ }^{\text {b) }}$ \\ Department of Chemistry and Chemical Biology, Harvard University, Cambridge, Massachusetts 02138, USA
}

(Received 3 November 2011; accepted 28 November 2011; published online 9 January 2012)

\begin{abstract}
Modeling of electronic structure of molecules in electrostatic environments is of considerable relevance for surface-enhanced spectroscopy and molecular electronics. We have developed and implemented a novel approach to the molecular electronic structure in arbitrary electrostatic environments that is compatible with standard quantum chemical methods and can be applied to mediumsized and large molecules. The scheme denoted CheESE (chemistry in electrostatic environments) is based on the description of molecular electronic structure subject to a boundary condition on the system/environment interface. Thus, it is particularly suited to study molecules on metallic surfaces. The proposed model is capable of describing both electrostatic effects near nanostructured metallic surfaces and image-charge effects. We present an implementation of the CheESE model as a library module and show example applications to neutral and negatively charged molecules. (C) 2012 American Institute of Physics. [doi:10.1063/1.3670417]
\end{abstract}

\section{INTRODUCTION}

Tremendous progress in the development and implementation of quantum chemistry methods has enabled $a b$ initio calculations of electronic structure, properties, and even dynamics to be performed on large-scale molecular systems containing many thousands of atoms. Despite these exciting developments in basic theory, fast numerical algorithms, and novel hardware utilization, ${ }^{1-5}$ it is not yet possible to routinely simulate such large systems using readily available computer resources. However, emerging nano-scale simulation challenges involving, for example, large biomolecular aggregates or nanotechnology devices are increasing the demand for firstprinciples methods that can deliver this performance. On the other hand, many of the problems of interest are inherently multi-scale and there is often no need to model all parts of the system at the full $a b$ initio level of theory.

In this work, we present the theory, implementation, and application of a simple, electrostatics-based approach for a specific class of multi-scale problems involving molecules at metallic interfaces of arbitrary shape. These systems are particularly relevant to the simulation of molecular nano-scale devices. ${ }^{6-8}$ Since molecular dimensions are in the range of $0.1-1 \mathrm{~nm}$, thus at least two orders of magnitude smaller than typical nanoparticles, we can identify the molecule as the system, while the nanoparticle is considered the environment. By treating only the system at the atomistic level of theory, we can recover the most important physics at a significantly reduced computational cost. Our goal is to provide a framework for describing interactions of molecules with complex nano-scale environments. This framework will be useful for studying interface phenomena that determine the properties and function of nano-scale devices, both in the absence and

\footnotetext{
a) Present address: Department of Chemical and Biomolecular Engineering and Department of Chemistry, Johns Hopkins University, Baltimore, Maryland 21218, USA.

b)Electronic mail: aspuru@chemistry.harvard.edu.
}

in the presence of an external bias. Our particular interest is in modeling surface-enhanced spectroscopies, ${ }^{9-11}$ especially surface-enhanced Raman scattering (SERS). ${ }^{9}{ }^{11-13}$ The last decade has seen surface-enhanced analytical methods become very useful tools for molecular detection as well as important probes into surface properties of metallic nanoparticles. Furthermore, the study of molecular properties under an external bias provided within the proposed framework is naturally connected to electron transport in nanostructures. ${ }^{14-16}$ Understanding of electron transport on the nano-scale is a crucial prerequisite for molecular electronics. Other applications where molecules interact non-trivially with a metallic surface also include surface-enhanced fluorescence ${ }^{17}$ and single-molecule DNA sequencing. ${ }^{18}$

The idea of partitioning a large system into subsystems to aid computation or understanding stretches back to the early works of Löwdin ${ }^{19}$ and McWeeny, ${ }^{20}$ where they introduced a rigorous mathematical framework for separating (localized) degrees of freedom in large-scale electronic structure calculations. Recently, there has been considerable interest in a practical, simplified version of these ideas known as the fragment molecular orbital (FMO) method ${ }^{21-24}$ which starts with the assumption that there are localized groups of electrons which interact with the other groups (to first order) only classically. A cluster expansion is used to correct for the many-body interaction energies after the fragment wavefunctions have converged. However, all fragments are treated at the same level of theory, so the method does not reflect the multi-scale nature of many interesting problems.

In contrast, the most common methods to treat very large chemical systems over the past two decades have focused on models where there is a natural division into typically two components, a system and an environment, treated at different levels of approximation. These models have a strong physical motivation and are often more heuristic in nature. Typically, these models aim to describe the effects of a complex environment on a localized chemical process, 
for example, solvation effects on molecular properties or the mechanisms of enzyme activity. In practice, there are essentially two schools of thought: the continuum models and the discrete models. The former are exemplified by the polarizable continuum model (PCM) ${ }^{25,26}$ and conductor-like screening model (COSMO). ${ }^{27,28}$ In these models, solvation is described by a single bulk component, characterized by a dielectric or screened conductor, rather than explicit solvent molecules. The discrete approach maintains an explicit treatment of the environment, but simplifies the calculation by avoiding the full treatment of all electronic degrees of freedom. The combined quantum-mechanical and molecular mechanics (QM/MM) models exemplify this approach. ${ }^{29-31}$ A fundamental issue with all of these methods, including the FMO, is how to treat the junctions between the components, especially when they cleave chemical bonds. Attempts have been made to put this on a more mathematical footing, but the pragmatic, more ad hoc approaches remain most popular.

Interactions between molecules and metallic surfaces include both an electromagnetic component ${ }^{32-34}$ and contributions from chemical bonding. ${ }^{35-38}$ The traditional approach to these systems has employed classical electrodynamics, in particular finite-difference time-domain (FDTD) methods, ${ }^{39,40}$ which can treat the strong, inhomogeneous electric fields present in nanostructures. The plasmonic properties of the metallic material are represented by its dielectric function. The metal-molecule interaction then amounts to the polarization of the molecule in the near-field of the nanostructure, while the back-polarization of the metal is neglected. Recent developments aim to integrate molecular electronic structure with an atomistic model of the plasmonic material. ${ }^{41-45} \mathrm{On}$ the other end of the method spectrum, continuum solvation models have found application to molecular electronic structure on metallic surfaces. ${ }^{46,47}$ Here, the metal-molecule interaction is derived from the mutual polarization of the neutral metallic surface and the electron density of the molecule.

As we show in detail below, the model proposed in this work encompasses both the strong-field and the zerofield, solvation-like regime as limiting cases and effectively interpolates between them. It is based on an electrostatic model, in which the metallic surface acts as a boundary condition for the electrostatic interactions within the molecule. As a consequence, we have to restrict our focus to situations where there is a clear system component that has minimal overlap with the environment, and for which there is a natural specification of the electrostatic potential at the system-environment interface. We argue that this is approximately the case in many nano-electronic devices. Recently, our group published a parallel paper ${ }^{48}$ where we used the same model, implemented inside the OCTOPUS ${ }^{49}$ code, to study the stabilization of the benzene anion between two charged plates. Our intentions in this paper are therefore threefold: first, to describe the theoretical background of the model in more detail; second, to explore a systematic set of test applications; and third, to meet the technical challenge of implementing the framework as a simple library that can be used in conjunction with conventional electronic structure codes, which expand the wavefunction or electron density in a Gaussian-type basis set. Gaussians give an extremely compact representation of the all-electron density, while allowing for the efficient analytical evaluation of two-electron Coulomb integrals. In contrast, OCTOPUS is a time-dependent density-functional theory (DFT) code which represents the electron density on a real-space grid. It computes the Hartree potential using a finite-difference Poisson solver, but this approach is rather computationally expensive for all-electron calculations. We therefore propose an algorithm for Hartree-Fock or DFT calculations which computes only a correction to the Fock or Kohn-Sham matrices, resulting from the interaction with the environment. The matrix elements for an isolated system are built using a standard Gaussian-based code in the usual way, and our library adds on contributions describing the environmental effects.

The paper is therefore organized as follows: in Sec. II, we characterize our physical model in detail, and provide a detailed derivation of the working equations for a DFT implementation, including a discussion of how we obtain the interaction potential and the associated matrix elements in an efficient manner. In Sec. III, we apply the method to study environment effects on the electronic structure of molecules both between grounded metal plates and under external bias. The effects of the external field and of the mutual polarization are explored for a neutral quadrupolar molecule (benzene), a neutral dipolar molecule (glycine zwitterion), and a charged molecule (benzene anion). Finally, we end with our conclusions and outlook for future work in Sec. IV.

\section{THEORY AND IMPLEMENTATION}

\section{A. Background}

To establish notation and to summarize connections to previous work, we begin by describing the electronic structure problem for systems in external electric fields, as well as in cavities within dielectric or metallic materials. Let us first consider the simple case of a molecular system subject to a time-independent external electric field,

$$
\mathbf{E}=-\nabla \Phi(\mathbf{r}),
$$

where $\Phi(\mathbf{r})$ is the electric scalar potential. In the special case of uniform fields,

$$
\Phi(\mathbf{r})=-\mathbf{E} \cdot \mathbf{r},
$$

where the potential origin is chosen to coincide with the coordinate origin. Thus the non-relativistic molecular electronic Hamiltonian for a system in a time-independent uniform electric field with Cartesian components $F_{\alpha}$ can be written as

$$
\hat{H}_{e}=\hat{H}_{e}^{(0)}-\sum_{\alpha} \hat{\mu}_{\alpha} F_{\alpha},
$$

where $\hat{H}_{e}^{(0)}$ is the unperturbed electronic Hamiltonian. The dipole moment operator, $\hat{\mu}$, is defined in the usual way,

$$
\hat{\mu}_{\alpha} \equiv-\sum_{i}^{N} r_{i \alpha}
$$


where the sum is over the $N$ electrons. More generally, for non-uniform fields, the perturbed Hamiltonian takes the form,

$$
\hat{H}_{e}=\hat{H}_{e}^{(0)}-\sum_{i}^{N} \Phi\left(\mathbf{r}_{i}\right)+C,
$$

where $C$ is an arbitrary constant depending on the choice of origin of the potential field, $\Phi(\mathbf{r})$. Of course, a change in $C$ does nothing more than shift the zero of energy, and has no effect on the electronic structure.

Many established quantum chemistry codes allow the treatment of Hamiltonians of one, or both, of these forms. Such functionality is relevant if one wants to find the electronic structure of a system embedded in an electrostatic environment, where the scalar potential, $\Phi(\mathbf{r})$, is explicitly known or easily computable. This is the case in the QM/MM and FDTD approaches, for example. On the other hand, an explicit model of the environment, even within an electrostatic approximation, may not always be available, or even desirable if the precise details of the surroundings are unimportant. This is the case for continuum solvation models such as PCM or COSMO, where the solute system is treated as a finite cavity (containing a molecule) within a bulk dielectric medium. In the PCM approach, the perturbing potential is defined implicitly in terms of the electrostatic boundary condition at the cavity-solvent interface, rather than by explicitly defined bulk "solvent" charges.

More concretely, in the apparent surface charge (ASC) formulation of the $\mathrm{PCM}^{25,26}$ a charge distribution, $\sigma(\mathbf{s})$, is identified at the dielectric interface, $\mathbf{s}$, which is produced by a discontinuity of the electric field across the boundary,

$$
\frac{\partial \Phi_{\text {in }}}{\partial \mathbf{n}}=\epsilon \frac{\partial \Phi_{\text {out }}}{\partial \mathbf{n}} .
$$

Here, $\epsilon$ is the dielectric constant of the solvent (the cavity is modeled in vacuo) and $\mathbf{n}$ denotes the outward unit normal from the cavity. According to elementary electrostatics for homogeneous linear dielectric media, $\sigma(\mathbf{s})$ is given by

$$
\sigma(\mathbf{s})=\frac{\epsilon-1}{4 \pi \epsilon}\left[\frac{\partial \Phi_{\text {sys }}}{\partial \mathbf{n}}+\frac{\partial \Phi_{\sigma}}{\partial \mathbf{n}}\right],
$$

where the total potential inside the cavity has been decomposed into two components: $\Phi_{\sigma}(\mathbf{r})$ is the potential due to the surface charge,

$$
\Phi_{\sigma}(\mathbf{r})=\int \frac{\sigma(\mathbf{s})}{|\mathbf{r}-\mathbf{s}|} \mathrm{d} \mathbf{s},
$$

and $\Phi_{\text {sys }}(\mathbf{r})$ is the solute potential,

$$
\Phi_{\text {sys }}(\mathbf{r})=\int \frac{\rho_{\text {sys }}\left(\mathbf{r}^{\prime}\right)}{\left|\mathbf{r}-\mathbf{r}^{\prime}\right|} \mathrm{d} \mathbf{r}^{\prime},
$$

where $\rho_{\text {sys }}(\mathbf{r})$ is the total (electronic and nuclear) charge density of the molecular system,

$$
\rho_{\text {sys }}(\mathbf{r})=\rho_{\text {sys }}^{\text {nuc }}(\mathbf{r})-\rho_{\text {sys }}^{\text {elec }}(\mathbf{r}),
$$

and we have introduced a minus sign for the electron charge. Self-consistent solution of Eqs. (7) and (8) with respect to $\sigma(\mathbf{s})$ and $\Phi_{\sigma}(\mathbf{r})$ for a given $\Phi_{\text {sys }}(\mathbf{r})$ is the central task in the ASC method. In turn, $\Phi_{\text {sys }}(\mathbf{r})$ may be obtained self-consistently with $\Phi_{\sigma}(\mathbf{r})$ from a quantum mechanical solution of the perturbed Hamiltonian,

$$
\hat{H}_{e}=\hat{H}_{e}^{(0)}-\sum_{i}^{N} \Phi_{\sigma}\left(\mathbf{r}_{i}\right) .
$$

In this way, the PCM approach models the electrostatic effect of the environment in a simple way without recourse to detailed atomistic knowledge of the solvent composition.

The technical challenge in the PCM/ASC approach stems from the fact that neither $\sigma(\mathbf{s})$ nor $\Phi_{\sigma}(\mathbf{s})$ are known a priori, and are only implicitly defined by the condition of selfconsistency in Eqs. (7) and (8). The boundary conditions are considerably simplified, however, when the solvent is modeled as a conductor (i.e., in the limit $\epsilon \rightarrow \infty$ ). In this case, the total potential at the solute/solvent interface must be a constant; indeed, it must be zero if the solvent extends to infinity. Then $\Phi_{\sigma}(\mathbf{r})$ is explicitly defined as a solution of Laplace's equation inside the cavity under Dirichlet boundary conditions,

$$
\begin{aligned}
\nabla^{2} \Phi_{\sigma}(\mathbf{r}) & =0 \quad \forall \mathbf{r} \in \text { cavity } \\
\Phi_{\sigma}(\mathbf{s}) & =-\Phi_{\text {sys }}(\mathbf{s}) .
\end{aligned}
$$

Unlike in the PCM/ASC algorithm, $\Phi_{\sigma}(\mathbf{r})$ can now be computed directly without any knowledge of $\sigma(\mathbf{s})$. Moreover, a good approximation to the true potential produced by (large) finite $\epsilon$ can also be obtained by rescaling $\Phi_{\sigma}(\mathbf{r})$ by a simple constant, $(\epsilon-1) / \epsilon$. This simplification (although not usually expressed this way) forms the basis of a wellknown approximation to the PCM, known as COSMO. ${ }^{27}$ PCM models have been previously applied to study electronic structure of molecules at metallic surfaces. ${ }^{46,47}$ In the following, we draw on all of these ideas to develop our model.

\section{B. The CheESE model}

In this work, we appeal to the above system-environment models with a different application focus in mind: namely, the simulation of molecules in electrostatic nano-environments where there is a natural and simple specification of the boundary conditions. We refer to our model as CheESE (chemistry in electrostatic environments). An implementation of the CheESE scheme in a real-space density functional code OCTOPUS was presented in a parallel paper. ${ }^{48}$ The new code presented here is implemented as a stand-alone library that can be easily interfaced to quantum chemistry packages employing Gaussian-type basis sets (see more details below). As in the PCM, we partition our problem into two components, analogous to solute and solvent, which we call system and environment, respectively. We then propose an effective electronic Hamiltonian for the system which includes an additional one-electron operator to describe the electrostatic effects of the environment. Thus our model has 3 key assumptions: 
1. We assume that system and environment degrees of freedom are uncoupled beyond the classical electrostatic interaction.

2. The system charge density is completely enclosed in the system volume, $\Omega_{\text {sys }}$; more precisely, there is an exponentially small overlap with the environment charge density.

3. The physics of the application provides a natural specification of the total electrostatic potential, $\Phi_{\text {fixed }}(\mathbf{s})$, on the system/environment interface, $\mathbf{s}$, which is known a priori.

Our model therefore differs from the solvation models discussed above in some important ways, even though they share the common physics of including only electrostatic effects of the environment. Specifically, we make no explicit appeal to the physical form of the environment, such as the assumption of a linear dielectric or conductor etc. Instead, we simply model the environment as a sea of charges that can be rearranged without any self-energy penalty such as to satisfy the boundary condition, $\Phi_{\text {fixed }}(\mathbf{s})$, which is known from the outset.

More formally, we can write down an effective Hamiltonian for the system, $\hat{H}^{\text {eff }}$, as a sum of two operators: the non-relativistic Hamiltonian of the isolated system, $\hat{H}^{(0)}$, and an environment potential, $\Phi_{\text {env }}(\mathbf{r})$,

$$
\hat{H}^{\mathrm{eff}}=\hat{H}^{(0)}-\sum_{i}^{N} \Phi_{\mathrm{env}}\left(\mathbf{r}_{i}\right)+\sum_{k}^{M} Z_{k} \Phi_{\mathrm{env}}\left(\mathbf{R}_{k}\right)
$$

Here, $\left\{\mathbf{r}_{i}\right\}$ and $\left\{\mathbf{R}_{k}\right\}$ are the coordinates of the system's $N$ electrons and $M$ nuclei (with charges $Z_{k}$ ), respectively. Formally, we have

$$
\Phi_{\mathrm{env}}(\mathbf{r})=\int \frac{\rho_{\mathrm{env}}\left(\mathbf{r}^{\prime}\right)}{\left|\mathbf{r}-\mathbf{r}^{\prime}\right|} \mathrm{d} \mathbf{r}^{\prime},
$$

in terms of the unknown environment charge density, $\rho_{\text {env }}(\mathbf{r})$, but according to assumptions 2 and 3 of the CheESE model it follows that the potential, $\Phi_{\text {env }}(\mathbf{r})$, inside the system volume is also a solution of Laplace's equation,

$$
\nabla^{2} \Phi_{\text {env }}(\mathbf{r})=0 \quad \forall \mathbf{r} \in \Omega_{\text {sys }},
$$

under the Dirichlet boundary condition,

$$
\Phi_{\text {env }}(\mathbf{s})=\Phi_{\text {fixed }}(\mathbf{s})-\Phi_{\text {sys }}(\mathbf{s}) .
$$

Note that $\Phi_{\text {sys }}(\mathbf{r})$ is the total potential due to the system's electrons and nuclei, as in Eqs. (9) and (10). For a given $\Phi_{\text {sys }}(\mathbf{r})$ and specified $\Phi_{\text {fixed }}(\mathbf{r})$, this boundary condition is known, and hence $\Phi_{\text {env }}(\mathbf{r})$ can be determined directly and uniquely without any explicit knowledge of $\rho_{\text {env }}(\mathbf{r})$. Our model implies that changes in $\rho_{\text {sys }}(\mathbf{r})$ will induce changes in $\rho_{\text {env }}(\mathbf{r})$ in order to maintain the constraint in Eq. (16), but physically we are not concerned with how this is achieved in detail.

Therefore, within the usual Born-Oppenheimer approximation, we define our model in terms of the ground state solution to the time-independent electronic Schrödinger equation,

$$
\hat{H}_{\mathrm{e}}^{\mathrm{eff}} \Psi\left(\left\{\mathbf{r}_{i}\right\} ;\left\{\mathbf{R}_{k}\right\}\right)=E\left(\left\{\mathbf{R}_{k}\right\}\right) \Psi\left(\left\{\mathbf{r}_{i}\right\} ;\left\{\mathbf{R}_{k}\right\}\right),
$$

where $\hat{H}_{\mathrm{e}}^{\text {eff }}$ is given by

$$
\begin{aligned}
\hat{H}_{\mathrm{e}}^{\mathrm{eff}}= & \sum_{i}^{N}-\frac{1}{2} \nabla_{i}^{2}+\sum_{i>j}^{N} \frac{1}{\left|\mathbf{r}_{i}-\mathbf{r}_{j}\right|} \\
& -\sum_{i}^{N} v_{\mathrm{nuc}}\left(\mathbf{r}_{i}\right)-\sum_{i}^{N} \Phi_{\mathrm{env}}\left(\mathbf{r}_{i}\right) .
\end{aligned}
$$

Here, we have used atomic units and $v_{\text {nuc }}(\mathbf{r})$ is the external potential due to the system's nuclei, defined in the usual way. We stress here that $\Phi_{\text {env }}(\mathbf{r})$ is itself an implicit function of the charge density of the system (which is an issue we will return to). Throughout the results (Sec. III), the energies we quote will be approximations to the total system energy defined by

$$
E_{\mathrm{tot}}^{\mathrm{sys}}=\left\langle\Psi\left|\hat{H}_{\mathrm{e}}^{\mathrm{eff}}\right| \Psi\right\rangle+\int \rho_{\mathrm{sys}}^{\mathrm{nuc}}(\mathbf{r}) \Phi_{\mathrm{env}}(\mathbf{r}) \mathrm{d} \mathbf{r}+U_{\mathrm{NN}},
$$

where we have included the interaction of the nuclear charge density, $\rho_{\text {sys }}^{\text {nuc }}(\mathbf{r})$, with the environment, in addition to the usual nuclear self-repulsion energy of an isolated system,

$$
U_{\mathrm{NN}}=\sum_{k>k^{\prime}}^{M} \frac{Z_{k} Z_{k^{\prime}}}{\left|\mathbf{R}_{k}-\mathbf{R}_{k^{\prime}}\right|} .
$$

Ultimately, one may ask what is physically being lost when we employ this model compared to a full ab initio calculation of the supersystem. The assumption that the system and environment have negligible overlap, however, makes the electrostatic approximation less dramatic than otherwise. For example, at the mean-field Hartree-Fock level of theory, there are no exchange interactions between non-overlapping subsystems, and the classical Coulomb interaction between subsystems suffices. In the case of generalized-gradient approximation density-functional theory, there are neither exchange nor correlation contributions between the system and environment, if non-overlapping. However, higher-order correlations obtained in wavefunction methods beyond Hartree-Fock exchange, such as dispersion, could be significant and our model of course neglects these. Nevertheless, such contributions can be expected to be much smaller than the dominant electrostatic interactions.

We mention one final issue in passing, namely, that we are neglecting any energy changes associated with the rearrangement of charge in the environment. In our parallel paper, ${ }^{48}$ where we also explore the stability of the benzene anion between two metallic plates, we estimate the energy required to charge the plates with induced charge. However, this energy is computed after the system's electron density has converged and does not influence the solution of the system Hamiltonian. A consideration of such effects could be included in further studies.

\section{Interpreting the environment potential}

We have found it useful when interpreting our results to conceptually partition the environment potential into two components,

$$
\Phi_{\text {env }}(\mathbf{r})=\Phi_{\text {image }}(\mathbf{r})+\Phi_{\text {static }}(\mathbf{r})
$$


where the linearity of the Laplace equation allows us to define each component individually,

$$
\begin{array}{cc}
\nabla^{2} \Phi_{\text {image }}(\mathbf{r})=0 ; & \Phi_{\text {image }}(\mathbf{s})=-\Phi_{\text {sys }}(\mathbf{s}), \\
\nabla^{2} \Phi_{\text {static }}(\mathbf{r})=0 ; & \Phi_{\text {static }}(\mathbf{s})=\Phi_{\text {fixed }}(\mathbf{s}) .
\end{array}
$$

In this way, we can identify a component, $\Phi_{\text {static }}(\mathbf{r})$, which is static in the sense that it represents an external potential that is completely independent of the molecule and exists solely to satisfy the fixed boundary condition, $\Phi_{\text {fixed }}(\mathbf{r})$. The remaining component, $\Phi_{\text {image }}(\mathbf{r})$, is seen to act like the potential of the induced charge (or image charge) on a grounded conductor. That is, it reflects the potential of the molecule, and adjusts such that the total potential, $\Phi_{\text {sys }}(\mathbf{s})+\Phi_{\text {image }}(\mathbf{s})$, vanishes on the boundary. In fact, $\Phi_{\text {image }}(\mathbf{r})$ is precisely the potential one would obtain in the COSMO solvation model summarized above, neglecting the final scaling by $(\epsilon-1) / \epsilon$ and assuming the same cavity geometry.

Below, we shall report our test applications with this decomposition in mind. In particular, we define the energy,

$$
E_{\text {static }}=E_{\text {tot }}^{\text {sys }}\left[\Phi_{\text {image }} \equiv 0\right]-E_{\text {tot }}^{\text {sys }}\left[\Phi_{\text {env }} \equiv 0\right],
$$

as the SCF energy difference between the isolated system and the SCF energy when applying a static field with no image charge effects included (i.e., a regular finite-field calculation). In turn, we also define,

$$
E_{\text {image }}=E_{\text {tot }}^{\text {sys }}-E_{\text {tot }}^{\text {sys }}\left[\Phi_{\text {image }} \equiv 0\right],
$$

as the SCF energy difference between a full CheESE calculation and a simple static field calculation. Note that $E_{\text {image }}$ is not strictly the same as $\int \rho_{\text {sys }}(\mathbf{r}) \Phi_{\text {image }}(\mathbf{r}) \mathrm{d} \mathbf{r}$, as extracted from a full CheESE calculation, since the image charges will perturb the system's electronic charge density away from the static field solution.

\section{Solving the nonlinear Schrödinger equation}

Having defined our model in terms of an effective Hamiltonian, we now discuss how to solve the associated Schrödinger equation (17). In contrast to the usual case for an isolated system, the equation is now formally nonlinear because the environment potential, $\Phi_{\text {env }}(\mathbf{r})$, is a function of $\rho_{\text {sys }}(\mathbf{r})$ due to the constraint in Eq. (16). An intuitive way to proceed, however, is to make a comparison with the wellknown Hartree-Fock (HF) or Kohn-Sham (KS) DFT approximations. For isolated systems, both of these approaches construct an approximate solution to the many-body problem with the explicit solution of only one-electron equations. One price to be paid for this great simplification is the sacrifice of linearity for nonlinearity. That is, the HF or KS equations are nonlinear and the iterative procedure usually employed to solve them self-consistently is very familiar.

In exactly the same way, we can consider a self-consistent procedure for the solution of the Schrödinger equation with the CheESE Hamiltonian. That is, we start by making an initial guess for the ground state density, $\rho_{\text {sys }}^{(0)}(\mathbf{r})$, and solve the Laplace equations (15) and (16) for $\Phi_{\mathrm{env}}^{(0)}(\mathbf{r})$, where $\Phi_{\mathrm{sys}}^{(0)}(\mathbf{s})$ on the boundary is computed from $\rho_{\text {sys }}^{(0)}(\mathbf{r})$. We may then construct the CheESE many-body Hamiltonian, $\hat{H}_{\mathrm{e}}^{\text {eff }}$, from $\Phi_{\mathrm{env}}^{(0)}(\mathbf{r})$ in a manner completely analogous to the construction of the Fock or Kohn-Sham matrix at each iterative cycle. Next, we can solve the Schrödinger equation with any approximate method we choose to obtain an improved ground state density and potential. The cycle is repeated with a new $\rho_{\text {sys }}^{(k)}(\mathbf{r})$, $\Phi_{\text {sys }}^{(k)}(\mathbf{r})$, and $\Phi_{\text {env }}^{(k)}(\mathbf{r})$ at the $k$ th iteration, and continued until self-consistency.

Although the above algorithm is general for any electronic structure method, we only use KS-DFT for our calculations here. Thus at the $k$ th iteration, we have a density, $\rho_{\text {sys }}^{(k)}(\mathbf{r})$, a potential, $\Phi_{\mathrm{env}}^{(k)}(\mathbf{r})$, and the KS equations take the form

$$
\hat{f} \phi_{i}(\mathbf{r})=\epsilon_{i} \phi_{i}(\mathbf{r}),
$$

where the modified KS operator is simply

$$
\hat{f}(\mathbf{r})=\hat{f}^{(0)}(\mathbf{r})-\Phi_{\mathrm{env}}^{(k)}(\mathbf{r}),
$$

and $\hat{f}^{(0)}(\mathbf{r})$ is the conventional operator for the isolated system. (Note that we have dropped any $k$-iteration suffices on the $\hat{f}$ operators.) On solving the KS equations, the total energy of the system is given by

$$
E_{\mathrm{tot},(k+1)}^{\mathrm{sys}}=E_{0,(k+1)}^{\mathrm{DFT}}+\int \rho_{\mathrm{sys}}^{(k+1)}(\mathbf{r}) \Phi_{\mathrm{env}}^{(k)}(\mathbf{r}) \mathrm{d} \mathbf{r}+U_{\mathrm{NN}},
$$

where $E_{0,(k+1)}^{\mathrm{DFT}}$ is the usual DFT energy functional for the isolated system. When changes in $E_{\mathrm{tot}}^{\mathrm{sys}}$ between two successive $k$-iterations are less than a specified threshold, we may assume the supersystem is converged, otherwise another iteration should be performed.

So far, we have assumed that the many-body problem is solved completely at each $k$-iteration (within an approximate method) for a given $\Phi_{\text {env }}^{(k)}(\mathbf{r})$. In KS-DFT, however, the KS equations themselves are solved iteratively in a self-consistent field (SCF) approach, implying that the above algorithm has two, nested, iterative cycles, differentiated by the point at which $\Phi_{\text {env }}(\mathbf{r})$ is updated. This raises the possibility of updating $\Phi_{\text {env }}(\mathbf{r})$ at each KS iteration, instead of after the KS solution has converged, thus effectively merging the two iterative cycles. Aside for issues of numerical stability, at convergence of $\Phi_{\text {env }}(\mathbf{r})$ and $\rho_{\text {sys }}(\mathbf{r})$ the final result should be the same. In fact, in our CheESE implementation, we do update $\Phi_{\text {env }}(\mathbf{r})$ at each KS iteration within the direct inversion in the iterative subspace-accelerated ${ }^{50} \mathrm{KS}$-SCF. It is not actually obvious whether it is more efficient to (perhaps partially) converge $\rho_{\text {sys }}(\mathbf{r})$ within the KS cycle before updating $\Phi_{\text {env }}(\mathbf{r})$, or to update both $\rho_{\text {sys }}(\mathbf{r})$ and $\Phi_{\text {env }}(\mathbf{r})$ at each KS iteration. The answer probably depends on the relative cost of updating the CheESE potential compared to a regular KS iteration, as well as how the numerical stability affects the number of iterations. Here, we have chosen to update both at each KS iteration and have observed good convergence, but the efficiency question is left open for a future study.

On a technical point, we mention that the CheESE nonlinearity implies that the expectation value of the effective Hamiltonian, $\hat{H}_{\mathrm{e}}^{\text {eff }}$, is not minimized in general by the (normalized) ground-state wavefunction (eigenfunction of $\hat{H}_{\mathrm{e}}^{\text {eff }}$ ), except in the special case that $\Phi_{\text {env }}(\mathbf{r})$ is independent of $\rho_{\text {sys }}(\mathbf{r})$. In turn, this implies that care needs to be taken when 
deriving the Hartree-Fock or KS equations, which are based on a variational condition. In fact, we side-step the issue here, when we define $\Phi_{\text {env }}^{(k)}(\mathbf{r})$ to be independent of $\rho(\mathbf{r})$ at each $k$-iteration, and hence obtain the modified KS operator in Eq. (27) quite trivially. However, it turns out that we can write down an alternative variational functional, which is minimized by the ground-state density, and also yields the same KS equations when taking the functional derivative. More details are given in the Appendix.

\section{E. Obtaining the environment potential}

We now discuss our implementation for computing the environment potential $\Phi_{\text {env }}(\mathbf{r})$ and the matrix elements of the modified Kohn-Sham operator (27) in a Gaussian basis set. First, we emphasize that we have chosen to compute the effect of the environment as a correction term to the isolated system Hamiltonian. However, we could alternatively define a KS operator,

$$
\hat{f}(\mathbf{r})=-\frac{1}{2} \nabla^{2}+v_{\mathrm{xc}}(\mathbf{r})-\Phi_{\mathrm{es}}(\mathbf{r}),
$$

where $v_{\mathrm{xc}}(\mathbf{r})$ is the DFT exchange-correlation potential and $\Phi_{\mathrm{es}}(\mathbf{r})$ collects all the electrostatic effects,

$$
\Phi_{\mathrm{es}}(\mathbf{r})=v_{\mathrm{nuc}}(\mathbf{r})-\int \frac{\rho_{\mathrm{sys}}^{\mathrm{elec}}\left(\mathbf{r}^{\prime}\right)}{\left|\mathbf{r}-\mathbf{r}^{\prime}\right|} \mathrm{d} \mathbf{r}^{\prime}+\Phi_{\mathrm{env}}(\mathbf{r}) .
$$

Formally, $\Phi_{\mathrm{es}}(\mathbf{r})$ can be obtained in one shot from Poisson's equation,

$$
\nabla^{2} \Phi_{\mathrm{es}}(\mathbf{r})=-4 \pi \rho_{\mathrm{sys}}(\mathbf{r}) \quad \forall \mathbf{r} \in \Omega_{s},
$$

subject to the specified boundary condition

$$
\Phi_{\mathrm{es}}(\mathbf{s})=\Phi_{\text {fixed }}(\mathbf{s}) .
$$

This is the formalism used in the OCTOPUS implementation, as described in our parallel paper. ${ }^{48}$ However, the full potential, $\Phi_{\mathrm{es}}(\mathbf{r})$, has many undesirable properties from an algorithmic perspective. In particular, even if we subtract out $v_{\text {nuc }}(\mathbf{r})$, the potential is still highly "spiked" near the nuclei and is therefore very difficult to represent accurately and efficiently using a grid-based numerical solver. Instead, we exploit the properties of Gaussian basis functions to compute matrix elements over these "spiked" operators analytically, and thus only deal with the correction term, $\Phi_{\text {env }}(\mathbf{r})$, by numerical solution. Compared to the other potentials, $\Phi_{\text {env }}(\mathbf{r})$ must be smooth by virtue of being a solution to Laplace's equation, which makes this approach much more attractive computationally.

More specifically, we base our Laplace solver for Eqs. (15) and (16) on a uniform finite-element discretization, following our previous work in Ref. 51. The starting point is to expand the potential as

$$
\Phi_{\mathrm{env}}(\mathbf{r})=\sum_{i} c_{i} \xi_{i}(\mathbf{r})
$$

where $\xi_{i}(\mathbf{r})$ is a three-dimensional tensor product of $n$th order one-dimensional piecewise Lagrange interpolating polynomials assigned to the $i$ th grid point. In the $N$ th finite element
(FE), the function assigned to the $i$ th point is given by

$$
\xi_{k l m}(\mathbf{r})=\left\{\begin{array}{l}
L_{k}\left(x-X_{N}\right) \cdot L_{l}\left(y-Y_{N}\right) \cdot L_{m}\left(z-Z_{N}\right) \\
0 \sim \text { (the } i \text { th grid point) } \notin \text { (the } N \text { th FE) },
\end{array}\right.
$$

where we define

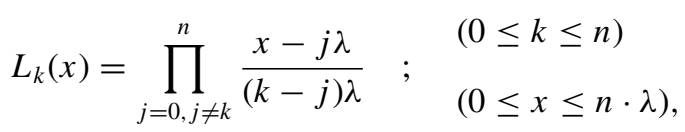

and $(k, l, m)$ is the local index of the $i$ th grid point in the $N$ th finite element; $\left(X_{N}, Y_{N}, Z_{N}\right)$ is the origin of the $N$ th finite element; and $n \cdot \lambda$ is the width of the finite elements. In all of our calculations, we use $3 \mathrm{rd}$ order polynomials. It is easy to see that the value of the potential at the $i$ th grid point is given by $c_{i}$. Substituting the expansion (33) into the Laplace equation and enforcing the appropriate boundary condition leads to a set of linear equations of the form

$$
\mathbf{A} \cdot \mathbf{c}=\mathbf{b},
$$

where

$$
A_{i j}=\int \nabla \xi_{i}(\mathbf{r}) \nabla \xi_{j}(\mathbf{r}) \mathrm{d} \mathbf{r},
$$

and the vector $\mathbf{b}$ describes the boundary condition. It is important to realize that the matrix $A_{i j}$ is extremely sparse and composed of relatively small, identical, and overlapping subblocks with $O\left(n^{6}\right)$ elements, where $n$ is the order of the polynomials. (Note that we have enforced continuity of the potential across element boundaries.) Exploiting this property, the full set of coefficients may be efficiently determined with an amount of work that scales linearly with the physical size of the solution domain for a given grid spacing. Here, we solve Eq. (35) iteratively using the conjugate gradient method.

To impose the boundary conditions in practice, we require an efficient method for evaluating $\Phi_{\text {sys }}(\mathbf{s})$ due to the system's electrons and nuclei. We achieve this using a multipole approximation previously given in detail in Ref. 51, which exploits the low scaling properties of the fast multipole method. Having obtained an explicit representation of the potential, the final task is to compute the matrix elements over Gaussian basis functions, and to update the free-space Kohn-Sham matrix. Fortunately, we can again exploit the properties of Gaussian functions and the polynomial form of the finite elements to do this in an analytical and simple way. Specifically, we use a modified form of the McMurchie-Davidson ${ }^{51,52}$ algorithm, which we have implemented for finite elements of arbitrary order.

Our code is designed as a stand-alone library, and in particular, the interface is extremely simple. The only information it requires is a description of the Gaussian basis set $\{\alpha\}$, nuclear charges and positions, the current atomic orbital density matrix, $D_{\alpha \beta}^{i}$, the fixed potential $\Phi_{\text {fixed }}(\mathbf{s})$, and of course the geometry of the solution domain, $\Omega_{\text {sys }}$. In return, the library will update the Kohn-Sham matrix elements, $F_{\alpha \beta}^{i}$, at each SCF cycle. Interfacing with an existing electronic structure code is therefore quite straightforward and requires only three calls to the CheESE library: (a) an initialization call, CheESE_init; (b) a call to update the Kohn-Sham matrix, CheESE_update; and (c) a final call to free the memory, 
CheESE_free. We summarize the structure of the code in Algorithm 1. For our prototype implementation, we have used the DALTON 2.0 package. ${ }^{53}$ Additional integration into other chemistry codes is encouraged and we expect to release the library on our group's website soon with a permissive LGPL license (http://www.gnu.org/copyleft/lesser.html).

ALGORITHM I. Structure of CheESE implementation.

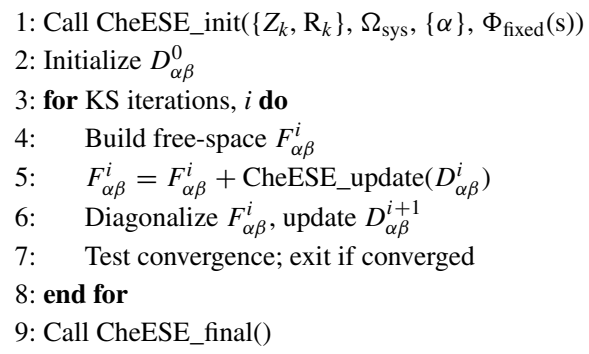

\section{BENCHMARKS AND APPLICATIONS}

\section{A. Computational details}

Our prototype code is restricted to the simple case of a cuboid-shaped system volume, $\Omega_{\text {sys }}$, with an arbitrary boundary potential, $\Phi_{\text {fixed }}(\mathbf{s})$. However, we have decided to choose a specific application focus: namely the simulation of a nanoelectronic device where we can imagine a molecule placed between two parallel electrodes or plates, held at fixed potentials, as shown in Fig. 1. This is similar to the studies we performed in our previous paper. ${ }^{48}$ Our intention is that the open-source library can be extended to other relevant geometries as required.

We describe in Sec. III B the precise geometries and potential functions we chose for $\Phi_{\text {fixed }}(\mathbf{s})$. In the finite-element Laplace solver, we used a value of $1.0 \mathrm{bohr}$ for the spacing, $\lambda$,

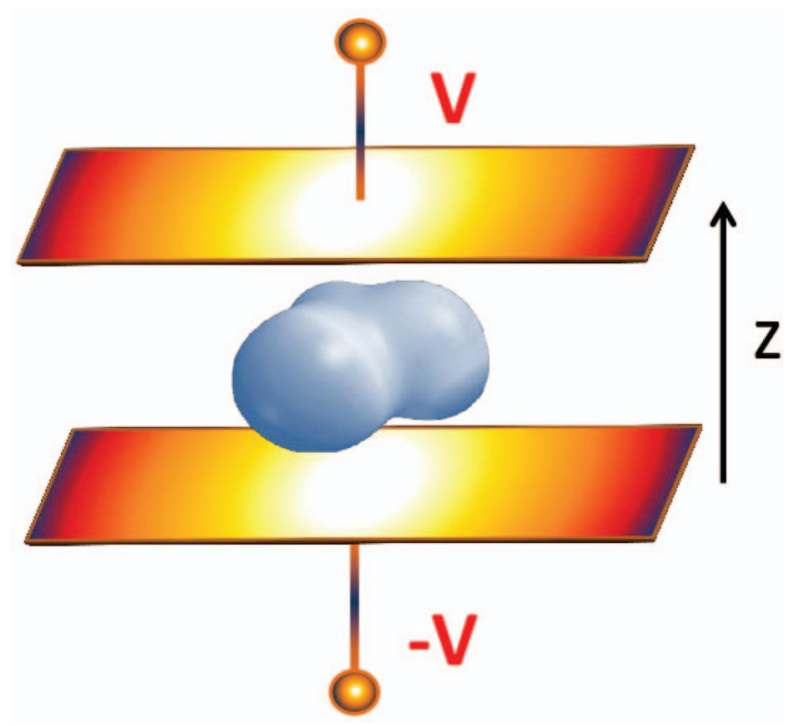

FIG. 1. Geometry of the CheESE model showing the direction of the applied potential, $\pm V$. We use a cuboid box, with the $x y$-plates representing the electrodes of a nano-device. The coordinate origin is equidistant from the two plates. in Eq. (34) for all the calculations in Sec. III C, and $2.0 \mathrm{bohr}$ for all the other calculations with non-zero $\Phi_{\text {fixed }}(\mathbf{s})$. We found that these values were sufficient to converge the results to the precision presented in all the figures. Note that the choice of $\lambda$ reflects the smoothness of the potential, $\Phi_{\text {env }}(\mathbf{r})$. Here we are able to use a relatively large $\lambda$, which results in a modest computational cost in the Laplace solver, especially for the smaller system volumes. Some example timings are given below.

All the calculations have been achieved by combining our library with the DALTON 2.0 quantum chemistry suite. ${ }^{53}$ As explained above, it is relatively simple to interface our code with any program using Gaussian-type basis sets. We have restricted our focus to DFT calculations using the popular B3LYP exchange-correlation functional. ${ }^{54}$ In addition, to avoid a detailed study of basis set effects, we elected to use the standard aug-cc-pVDZ basis for all calculations. This should be a reasonable choice for the level of accuracy we are expecting in this work, with the diffuse functions providing some flexibility for any spatial redistribution of the electron cloud under potential bias.

We have chosen to study the CheESE effect on three molecules with a similar size and number of electrons, but different symmetries: namely, (1) benzene (neutral, quadrupolar ground state), (2) benzene anion (charged, monopolar ground state), and (3) glycine zwitterion (neutral, dipolar ground state). The benzene and benzene anion geometries were optimized at the B3LYP/aug-cc-pVDZ level using DALTON. In all calculations, the benzene rings were placed in the $x y$-plane, with the centre of mass at the coordinate origin. The glycine geometry was taken from Ref. 55. The molecule was placed such that the amine group was at a positive $z$-coordinate, and rotated such that the electric dipole moment (with no external field) was parallel to the $z$-axis. The centre of mass was also placed at the origin.

\section{B. Specification of the boundary conditions}

As stated above, our intention is to model a molecule in a cuboid cavity representing a nano-device, with a potential bias applied across two surfaces. For our test calculations, we have therefore chosen a very simple form for the fixed boundary potential, defined as

$$
\begin{gathered}
\Phi_{\text {fixed }}\left(z_{\text {max }}\right)=V, \\
\Phi_{\text {fixed }}\left(z_{\text {min }}\right)=-V, \\
\Phi_{\text {fixed }}(\mathbf{s})=\frac{z}{z_{\max }} V,
\end{gathered}
$$

on the six sides of the cavity, which linearly interpolates the potential, $V$, from the top plate to the bottom plate. In all cases, the box is positioned in space such that the plates sit in the $x y$ plane and are equidistant from the coordinate origin and the centre of the cavity is at the coordinate origin [see Figure 1, (i.e., $z_{\max }=\left|z_{\min }\right|$.)]. In the following, we shall refer to $\Delta z$ as the plate separation,

$$
\Delta z \equiv z_{\max }-z_{\min }
$$

In most of the calculations, we chose plate dimensions of $108 \times 108$ bohr $^{2}$, but in some cases given below, a larger area 


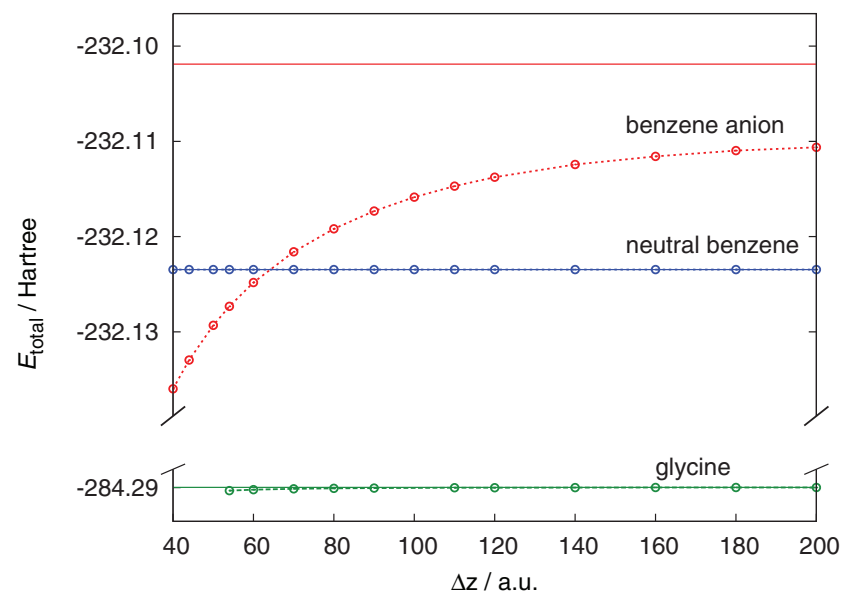

(a) Total energies

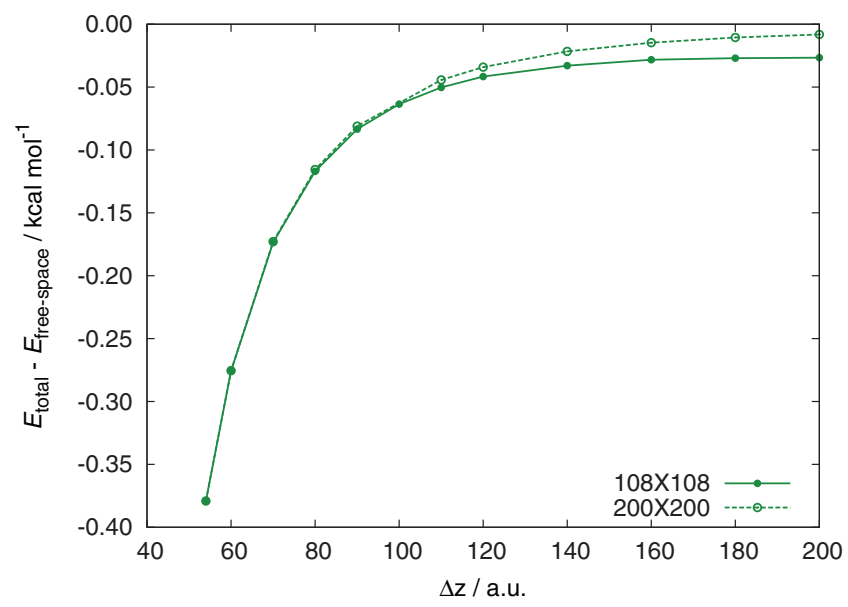

(c) Glycine (image effect)

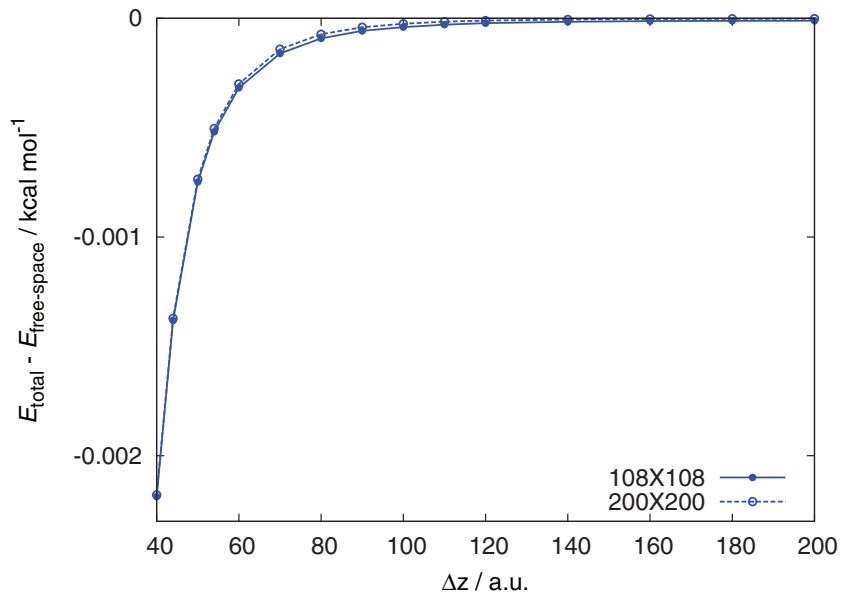

(b) Neutral benzene (image effect)

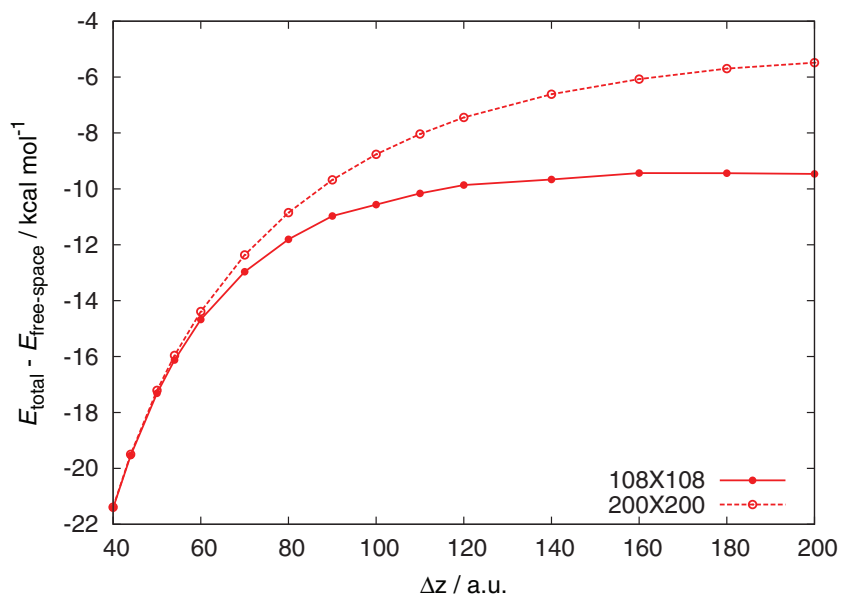

(d) Benzene anion (image effect)

FIG. 2. Stabilization of the benzene (blue lines), benzene anion (red lines), and glycine (green lines) molecules due to CheESE image charges in the case $\Phi_{\text {fixed }}(\mathbf{s})=0$ with varying $\Delta z$. Part (a) compares the total energies, $E_{\text {tot }}^{\text {sys }}$, shown as dotted lines, with the reference free-space energies, shown as solid lines, for an $x y$-plate area of $200 \times 200$ bohr $^{2}$. Parts (b)-(d) highlight the CheESE effect as the difference between $E_{\text {tot }}^{\text {sys }}$ and the free-space energy for (b) benzene, (c) glycine, and (d) benzene anion, with two choices of plate area.

of $200 \times 200$ bohr $^{2}$ was studied. As for the plate separation, we studied values of $\Delta z$ ranging from $40 \mathrm{bohr}$ to $200 \mathrm{bohr}$.

For all the calculations, we applied boundary potentials of no more then 1 a.u. in magnitude, which corresponds to approximately $27.2 \mathrm{~V}$. Thus for a plate separation of $54 \mathrm{bohr}$ (which is typical in our calculations), this amounts to an electric field of approximately $2 \mathrm{VA}^{-1}$ when the plates are held at \pm 1 a.u. This magnitude is similar to the field strengths previously studied by Choi et al. in their work on molecular orbital tuning, ${ }^{56,57}$ but is larger than the field strengths we explored in our previous paper. ${ }^{48}$

We note that this choice of fixed potential makes the calculation of $\Phi_{\text {static }}(\mathbf{r})$ rather trivial. Of course, this is the potential associated with a uniform electric field parallel to the $z$-axis, of magnitude $V / z_{\max }$. Indeed, $\Phi_{\text {static }}(\mathbf{r})$ can be accurately captured with only a single finite element in this case. Moreover, DALTON 2.0 already has the capability to compute Kohn-Sham matrix elements under such a uniform field. Therefore, the most interesting part of the calculation is the contribution of $\Phi_{\text {image }}(\mathbf{r})$ and this is the area we will focus on. Nevertheless, our code can already handle more complicated
$\Phi_{\text {static }}(\mathbf{r})$, so future applications will be aimed towards exploring this functionality in addition to the image charge effects.

Finally, we emphasize that the CheESE model requires negligible overlap of electron density between the system and environment. This was enforced in practice by simply choosing a geometry which ensured that no basis function tail had a significant value $\left(>10^{-10}\right)$ in the boundary region. This is a conservative constraint; a consideration of the density itself would be favourable in future work if a boundary closer to the molecular system was desired.

\section{Energy calculations with $\Phi_{\text {fixed }}(s)=0$}

For our test applications, we first explore the case where $\Phi_{\text {fixed }}(\mathbf{s})=0$; that is, $\Phi_{\text {static }}(\mathbf{s})=0$ so the Hamiltonian is only modified by the image charge effects contained in $\Phi_{\text {image }}(\mathbf{r})$. The image charge potential is given by Eq. (22) and hence depends explicitly on the total potential of the system at the boundary, $\Phi_{\text {sys }}(\mathbf{s})$. We can therefore expect quite different effects for our three molecules, reflecting the decay properties of $\Phi_{\text {sys }}(\mathbf{r})$ at long range. In Fig. 2, we show how the total en- 
ergy, $E_{\text {tot }}^{\text {sys }}$ in Eq. (28), of the three test molecules changes as a function of the plate separation, $\Delta z$, compared to the freespace energies. In Fig. 2(a), we compare the total energies for all three molecules with a plate area of $200 \times 200$ bohr $^{2}$ and varying $\Delta z$, while in Figs. 2(b)-2(d), we highlight the stabilization energy due to the image effects for each molecule individually, also including results for a smaller plate size of $108 \times 108$ bohr $^{2}$.

In these calculations, the stabilization energy relative to the free-space result reduces to $\Delta E=\int \rho_{\text {sys }}(\mathbf{r}) \Phi_{\text {image }}(\mathbf{r}) \mathrm{d} \mathbf{r}$ within an excellent approximation, since the electron density is hardly perturbed and there is no static field. $\Phi_{\text {image }}(\mathbf{r})$ is given by Eq. (22), so it will be larger if $\Phi_{\text {sys }}(\mathbf{s})$ is larger. Indeed, if $\Phi_{\text {sys }}(\mathbf{s})$ is a constant, this will be the uniform value of $\Phi_{\text {image }}(\mathbf{r})$ inside the cavity. However, note that even if $\Phi_{\text {image }}(\mathbf{r})$ is large, $\Delta E$ will vanish for charge-neutral molecules if $\Phi_{\text {image }}(\mathbf{r})$ is uniform because the electronic and nuclear contributions will exactly cancel. Therefore, especially for the charge-neutral systems, the size of $\Delta E$ depends not only on the magnitude of $\Phi_{\text {sys }}(\mathbf{s})$ but also on its inhomogeneity. The latter is a function of both the cavity geometry and the symmetry of the system's charge distribution.

Looking at Fig. 2, the first point to note is that all the systems are stabilized by the boundary effect. We can attempt to interpret these results by appealing to an image charge picture, and this is readily done for the benzene anion. In this case, the system generates a negative potential on the boundary. Hence, the boundary condition (22) implies that the anion must induce a distribution of positive image charge in the environment such that the total potential vanishes on the cavity surface. Naturally, this positive charge stabilizes the negative charge of the anion. However, there is a sensitive cancellation of electronic and nuclear contributions. For example, with $\Delta z=54$ bohr and the smaller $(108 \times 108)$ plates, the nuclear contribution, $\int \rho_{\text {sys }}^{\text {nuc }}(\mathbf{r}) \Phi_{\text {image }}(\mathbf{r}) \mathrm{d} \mathbf{r}$, is positive $(679 \mathrm{kcal} / \mathrm{mol})$, and is only just outweighed by its electronic counterpart, $\int \rho_{\text {sys }}^{\text {elec }}(\mathbf{r}) \Phi_{\text {image }}(\mathbf{r}) \mathrm{d} \mathbf{r}$, which is negative $(-695 \mathrm{kcal} / \mathrm{mol})$ leading to a net stabilization.

The stabilization energies of the glycine and neutral benzene systems are much smaller, however. Specifically, while the anion is stabilized by as much as $21 \mathrm{kcal} / \mathrm{mol}$, depending on $\Delta z$, the stabilization energy for the glycine zwitterion is about 50 times smaller, and for neutral benzene it's about four orders of magnitude smaller. This is not very surprising. To a good approximation, the cavity boundary sees the anion as a monopole, the glycine as a dipole, and the neutral benzene as a quadrupole source at the origin. The image charges in the environment therefore reflect these symmetries. As a result, $\Delta E$ is dominated by charge-charge interactions in the anion case, but only dipole-dipole interactions in the glycine case, for example. Since the latter decay more quickly with distance, it is not surprising that the stabilization energy is much smaller. More specifically, in the neutral benzene case, again for $\Delta z=54 \mathrm{bohr}$ and the smaller $(108 \times 108)$ plate size, the nuclear contribution to $\Delta E$ is only $1.6368 \mathrm{kcal} / \mathrm{mol}$ and the electronic component is -1.6373 $\mathrm{kcal} / \mathrm{mol}$. Overall, there is a net stabilization energy, but it is much smaller than for the anion. As a result, for plate separations less than about $65 \mathrm{bohr}$, we find that the anion is ac- tually more stable than neutral benzene, which is stabilized much less by the image effect. (In free-space, we find that the anion is about $14 \mathrm{kcal} / \mathrm{mol}$ less stable than the anion. The experimental result is about $26 \mathrm{kcal} / \mathrm{mol} .{ }^{58,59}$ Other examples of image stabilization from electrons interacting with their environments have been studied experimentally by Harris and co-workers; see, for example, Ref. 62 and references therein.) In the glycine case, under the same conditions, the nuclear contribution is $-2.5 \mathrm{kcal} / \mathrm{mol}$, and the electronic contribution is $2.1 \mathrm{kcal} / \mathrm{mol}$, giving a net stabilization which is much larger than in neutral benzene, even though the individual terms have the same order of magnitude. In fact, the balance of nuclear and electronic terms is subtle; for example, in glycine and neutral benzene, the nuclear term changes sign for some plate separations.

To further understand the relative values of $\Delta E$, we can also examine the size of $\Phi_{\text {sys }}(\mathbf{s})$, which directly reflects the magnitude of the resulting $\Phi_{\text {env }}(\mathbf{r})$. For example, in the case $\Delta z=60$ bohr and the smaller plate size, we observe $\Phi_{\text {sys }}(\mathbf{s}) /$ a.u. at the far corner of the system volume (54.0, 54.0, 30.0 ) to be $3.1 \times 10^{-6}, 2.3 \times 10^{-4}$, and $-1.2 \times 10^{-2}$ for the neutral benzene, glycine, and anion, respectively. Consistent with the size of $\Delta E$, we see that the anion potential is about four orders of magnitude larger than the neutral benzene potential.

It is also interesting to consider the decay behaviour of $\Delta E$ with plate separation, $\Delta z$. It is generally true that as the cavity, $\Omega_{\text {sys }}$, gets larger, $\Phi_{\text {sys }}(\mathbf{s})$ gets smaller, and the image charge effect is reduced. In the limit of an infinitely large cavity, $\Delta E$ is of course zero and the CheESE energy converges to the free-space energy. This effect is very clear for all the molecules in the figure, but we can also see that $\Delta E$ decays relatively more slowly for the anion than glycine, and neutral benzene shows the most rapid decay as $\Delta z$ increases. Again, this is due to the different multipole characteristics of the molecules and the decay rate of $\Phi_{\text {sys }}(\mathbf{s})$ with $\Delta z$. We have also shown the difference when using smaller or larger $x y$-plates, which is particularly significant for the anion. In this case, to a good approximation, $\Phi_{\text {sys }}(\mathbf{s})$ is spherically symmetric about the origin. Hence, when using the smaller $(108 \times 108)$ plates, the "edge effect" due to the finite plate areas, and the the potential on the sides of the box (i.e., $x z$ and $y z$ planes) becomes the limiting boundary condition as $\Delta z$ exceeds about 100 bohr. This difference is less pronounced in the glycine and neutral benzene cases due to the different charge distributions. With glycine, for example, the dipole is aligned along the $z$-axis, and we find that $\Phi_{\text {sys }}(\mathbf{s})$ on the $x y$-plates is approximately two orders of magnitude larger than on the $x z$ and $y z$ planes at equal distance; hence, changes in stabilization as $\Delta z$ varies are less affected by changes in the plate size.

\section{Energy calculations with $\Phi_{\text {fixed }}(s) \neq 0$}

Here we explore the situation with non-zero static field, $\Phi_{\text {static }}(\mathbf{r})$, in addition to the image charge effects. In particular, $\Phi_{\text {fixed }}(\mathbf{s})$ is given by Eq. (39), which describes a uniform external field applied along the $z$-axis. As before, we study the same three test molecules, and examine the change in $E_{\text {tot }}^{\text {sys }}$ as a function of the applied bias $V$. The results are presented in 


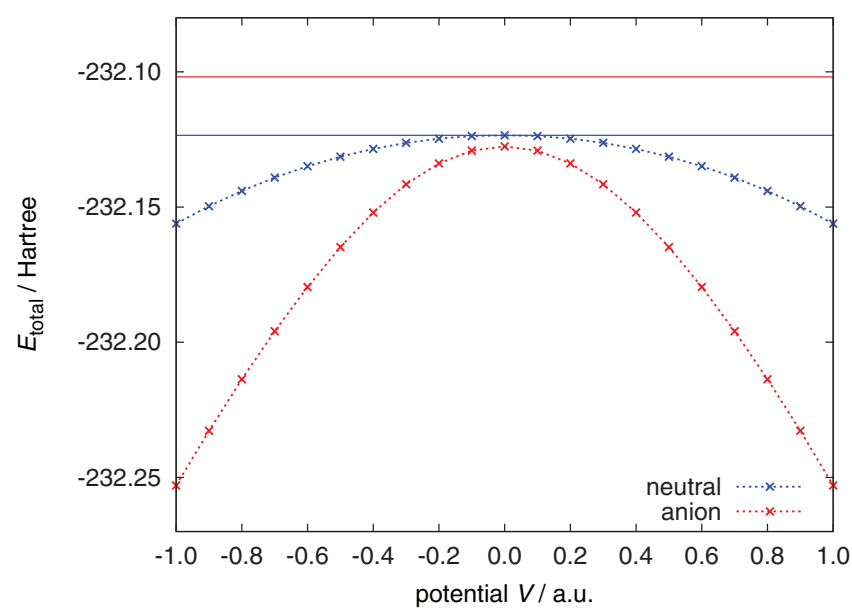

(a) Benzene (neutral and anion)

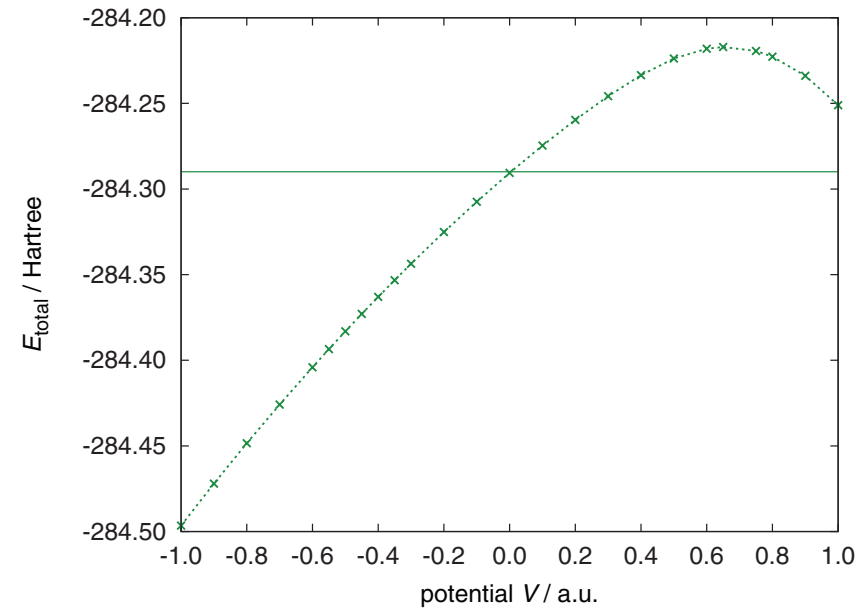

(b) Glycine

FIG. 3. Energies of neutral benzene (blue lines), benzene anion (red lines), and glycine (green lines), with an applied potential, $V$, as given in Eq. (39), an $x y$-plate area of $108 \times 108 \mathrm{bohr}^{2}$, and $\Delta z=54 \mathrm{bohr}$. The total CheESE energies, $E_{\mathrm{tot}}^{\text {sys }}$, are shown as dashed lines, and the reference free-space energies are shown as solid lines.

Figs. 3 and 4, where we have chosen to use the smaller 108 $\times 108$ bohr $^{2} x y$-plates and a $z$-spacing of $54 \mathrm{bohr}$ throughout. This implies an external field strength of approximately $2 \mathrm{VA}^{-1}$ when $V= \pm 1.0$ a.u. The behaviour of molecules under static external fields has been thoroughly studied in the literature, so the main purpose of this discussion is to examine the significance of the image charge effects. However, we will briefly summarize the main points of Fig. 3 first, which reports the total CheESE energy, $E_{\mathrm{tot}}^{\text {sys }}$, with varying $V$, and mostly reflects the static field effect.

Due to the choice of the applied potential, $\Phi_{\text {fixed }}(\mathbf{s})$, and choice of geometries, the neutral and anionic benzene results are symmetric with respect to sign changes in $V$, and in both cases the molecules are stabilized, with negative energy contributions from both the static field interaction and the image charge effects discussed in Sec. III C. As the field increases, the static field induces a larger electronic dipole and the stabilization increases, while the image charge contribution is only moderately increased. In contrast, the glycine molecule is not symmetric along the $z$-axis and we observe different energies for positive and negative $V$. In fact, for positive $V$, the static field has an unfavourable direction with respect to the molecule's net dipole moment and the system is destabilized by the static field, even though the image charge contribution actually remains negative. (Recall that the amine group is nearest the positive-z plate.) However, as the field gets stronger, the electron density is sufficiently distorted towards the upper plate that the dipole changes sign (at approximately $V=0.65$ a.u.) and we observe a turning point in the total energy curve.

The static field effects are therefore clearly important, but here we are most interested in the image charge contributions. Therefore, in Table I and Fig. 4, we highlight the individual contributions of the static and image charge potentials, $E_{\text {static }}$ and $E_{\text {image }}$, as defined in Eqs. (24) and (25). Looking at these results, it is clear that for neutral benzene and glycine the static field effect is much more significant than the image charge stabilization. Even for the smaller static field strengths $\left(\sim 0.2 \mathrm{~V}^{-1}\right), E_{\text {static }}$ is greater than $E_{\text {image }}$ by at least an order of magnitude in glycine, and two orders of magnitude in neutral benzene. For the benzene anion, however, the situation is different. In fact, for a field strength of $1 \mathrm{~V}^{-1}$, the image and static contributions are comparable. Moreover, in the weaker field, $\sim 0.2 \mathrm{~V}^{-1}$, the image charge stabilization is approximately $16 \mathrm{kcal} / \mathrm{mol}$, while the stabilization due to the static field is less than $1 \mathrm{kcal} / \mathrm{mol}$ : an order of magnitude smaller!

In addition to comparing the relative sizes of $E_{\text {static }}$ and $E_{\text {image }}$, it is also interesting to observe how $E_{\text {image }}$ depends on the applied potential, $V$. Looking at Fig. 4, we can see the expected symmetrical results for the benzene systems. Moreover, as $V$ increases, $E_{\text {image }}$ increases in magnitude. At zero $V$, the results are the same as shown in Fig. 2. As the bias is applied, the electron cloud is distorted and the image charge distribution changes from its approximate spherical symmetry. In fact, the change in the image charges is such as to reinforce the bias, $V$, which is already stabilizing the system. In the anion, however, the change in $E_{\text {image }}$ due to increased bias is less than $1 \%$ across the range of $V$ we study. In glycine, we see a non-symmetric change with $V$. Moreover, while $E_{\text {static }}$ is positive for $V>0, E_{\text {image }}$ is negative for all $V$, although in all cases it is quite small: less than $1 \mathrm{kcal} / \mathrm{mol}$ in magnitude. Note that the turning point in $E_{\text {image }}$ occurs at the same bias potential as the turning point in $E_{\text {static }}$, when the molecular dipole goes to zero. From our discussion in Sec. III C, this is perhaps not surprising. We know that the image charge potential depends on $\Phi_{\text {sys }}(\mathbf{s})$, and as the dipole tends to zero, we can expect this boundary potential to decrease as it depends on more rapidly decaying higher-order moments. Indeed, this is what we observe in the calculations.

\section{E. Molecular orbital tuning}

So far, we have looked at total molecular energies. However, as already mentioned, it is possible to shift the total energy without making any interesting changes to the electronic structure of the system. Indeed, the addition of a constant 


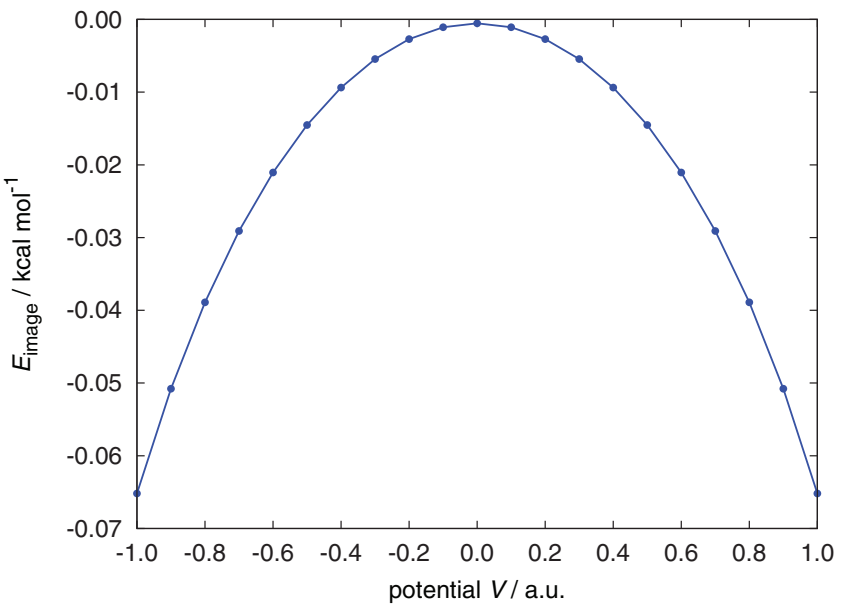

(a) Neutral benzene

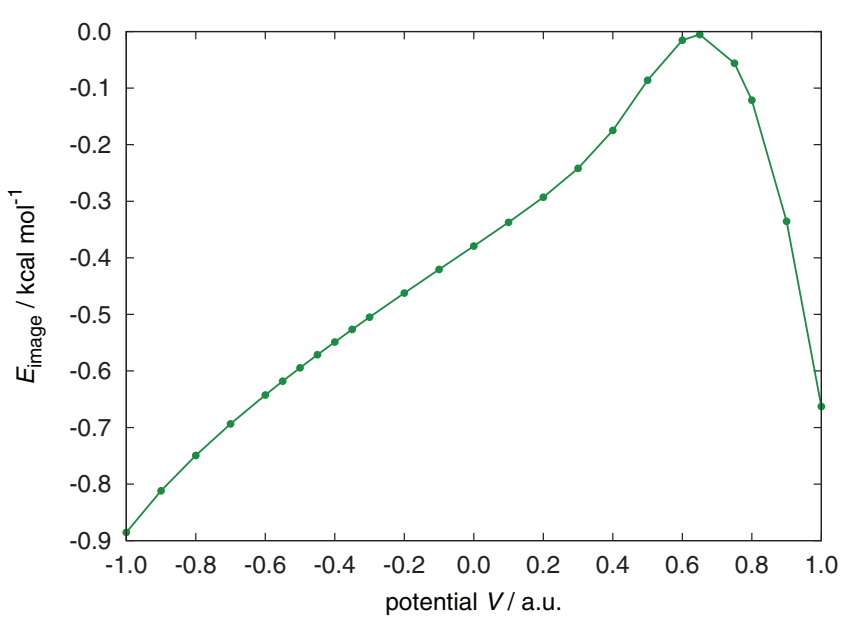

(b) Glycine

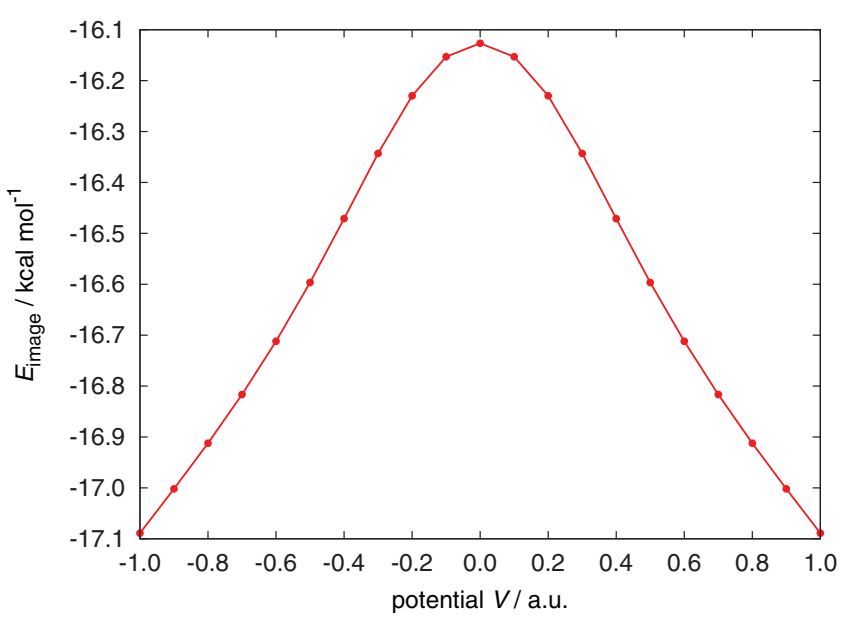

(c) Benzene anion

FIG. 4. Energy changes for different applied potentials when including image charge effects in addition to a static field. $E_{\text {image }}$, as given by Eq. (25), is shown for (a) neutral benzene, (b) glycine, and (c) benzene anion, using an $x y$-plate area of $108 \times 108 \mathrm{bohr}^{2}$, and $\Delta z=54$ bohr.

potential to the Kohn-Sham operator will shift all the orbital energies by that same constant, but leave the molecular orbitals themselves unchanged. In other words, it is important to observe if the orbital energies change in different ways. For
TABLE I. Energy differences in kcal/mol, according to Eqs. (24) and (25), giving the change, $E_{\text {static }}$, to the total energy on applying a static field with potential V/a.u., and the additional CheESE image charge contribution, $E_{\text {image }}$.

\begin{tabular}{|c|c|c|c|c|c|c|}
\hline \multirow[b]{2}{*}{$V$} & \multicolumn{2}{|c|}{$\mathrm{C}_{6} \mathrm{H}_{6}$} & \multicolumn{2}{|c|}{ Glycine } & \multicolumn{2}{|c|}{$\mathrm{C}_{6} \mathrm{H}_{6}^{-}$} \\
\hline & $E_{\text {static }}$ & $E_{\text {image }}$ & $E_{\text {static }}$ & $E_{\text {image }}$ & $E_{\text {static }}$ & $E_{\text {image }}$ \\
\hline-1.0 & -20.44 & -0.065 & -128.74 & -0.89 & -77.72 & -17.09 \\
\hline-0.5 & -4.94 & -0.015 & -57.87 & -0.59 & -22.89 & -16.60 \\
\hline-0.1 & -0.20 & -0.001 & -10.54 & -0.42 & -0.95 & -16.15 \\
\hline 0.1 & -0.20 & -0.001 & 9.97 & -0.34 & -0.95 & -16.15 \\
\hline 0.5 & -4.94 & -0.015 & 41.61 & -0.09 & -22.89 & -16.60 \\
\hline 1.0 & -20.44 & -0.065 & 25.08 & -0.66 & -77.72 & -17.09 \\
\hline
\end{tabular}

example, the HOMO (highest occupied molecular orbital) and LUMO (lowest unoccupied MO) orbital energies are significant in nano-electronic or electron transport studies. ${ }^{14-16,60}$ Above all, the HOMO-LUMO gap is an important property, and if the orbital energies are simply shifted by a constant, then the gap will obviously not change.

In Figs. 5 and 6, we therefore plot how the HOMO, LUMO, and HOMO-LUMO gap change when we apply the CheESE model. Figure 5 gives the energy profiles of the HOMO and LUMO with varying $V$ for the three molecules including all the image effects. Since the LUMO changes character as the orbitals cross in the neutral benzene case, we have shown the three lowest lying orbitals. This explains the kinks in the neutral benzene curves in Fig. 6. More precisely, Fig. 6(a) plots the difference between the HOMO-LUMO gap in an isolated system and when applying only a static field. Figure 6(b) plots the difference between the HOMO-LUMO gap computed by a full CheESE calculation (with bias potential $V$ ) and a calculation with only the static field.

The main message in Fig. 5 is that the orbital energies change significantly with applied field, thereby allowing the possibility of orbital "tuning." Moreover, due to greater changes in the LUMO compared to the HOMO, we see changes to the HOMO-LUMO gap of as much as $75 \mathrm{kcal} / \mathrm{mol}$ (e.g., glycine at large positive $V$ ) compared to free-space. Indeed, the glycine HOMO-LUMO gap reduces dramatically when the field is large and directed against the (free-space) dipole moment. The figure does not make clear the static and image charge contributions, but the details are somewhat complex. For example, in the benzene anion, the static field stabilizes the LUMO, but actually destabilizes the HOMO, while the (large, but fairly constant) image charge effect stabilizes both orbitals. In glycine, the static field stabilizes both HOMO and LUMO for all $V$, while the (small, but variable) image charge effect can either stabilize or destabilize the orbitals, depending on $V$.

On the other hand, we are often most interested in the gap, rather than the precise orbital energies. Figure 6 distinguishes the image charge and static contributions in this case. As expected, we see that the static field effect is generally more important than the image effect. In some cases, the energy change is two orders of magnitude more than that due to the image effect. Nevertheless, the image effect is not completely negligible, and modifies the gap by almost $1 \mathrm{kcal} / \mathrm{mol}$ in the glycine case when $V=-1.0$ a.u. 




(a) Neutral benzene

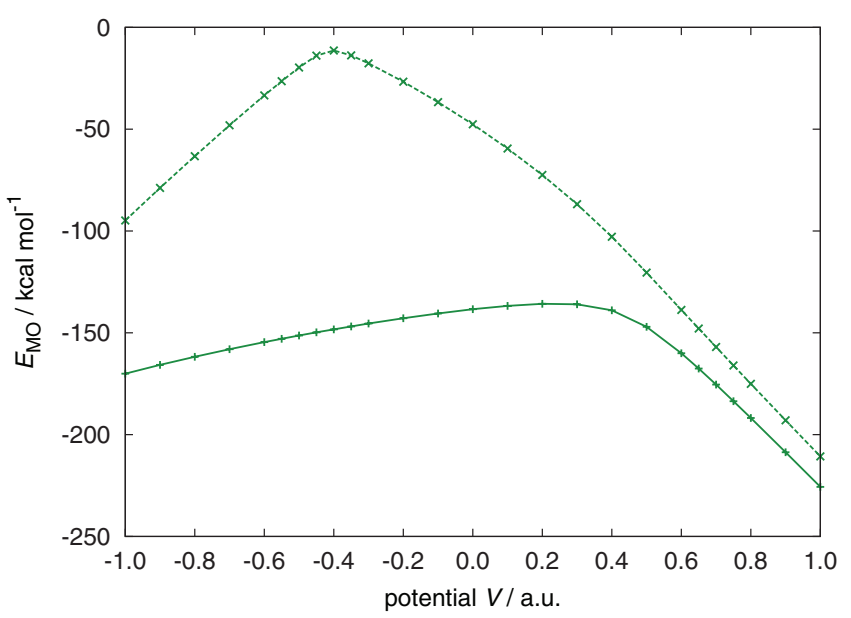

(b) Glycine

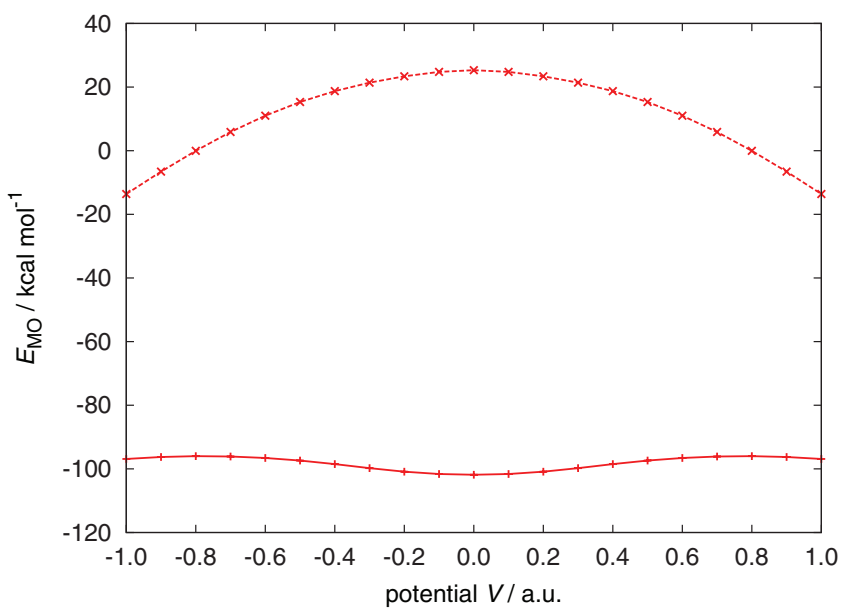

(c) Benzene anion

FIG. 5. Energy profiles of the lowest lying molecular orbitals of (a) neutral benzene, (b) glycine, and (c) benzene anion, with an applied potential, $V$, an $x y$-plate area of $108 \times 108 \mathrm{bohr}^{2}$, and $\Delta z=54 \mathrm{bohr}$. Since there are no relevant orbital crossings, only the HOMO and LUMO are shown for the anion and glycine.

Moreover, with zero static field, only the image charges produce a change, and in the glycine case the gap is increased by approximately $0.5 \mathrm{kcal} / \mathrm{mol}$ when $V=0$. Interestingly, when considering the gap, the image charge effects are most

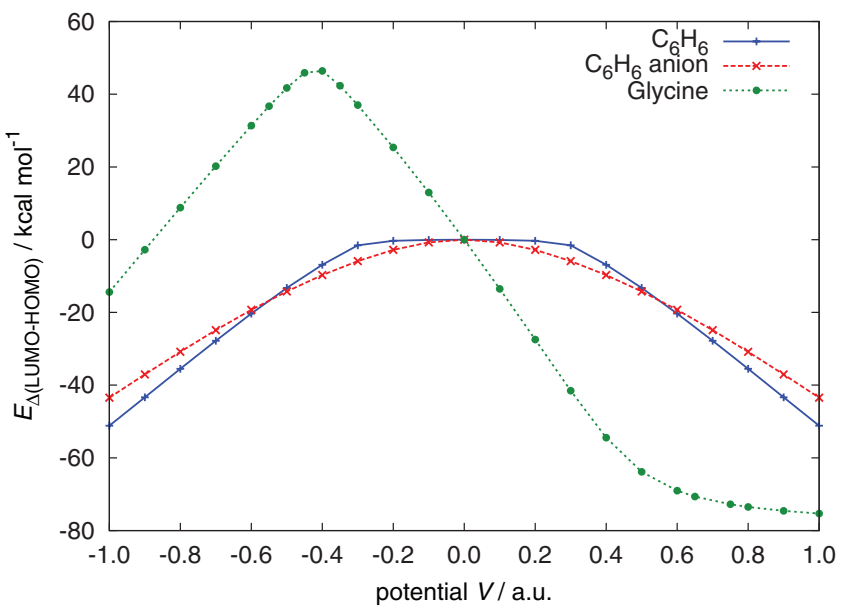

(a) Static field contributions

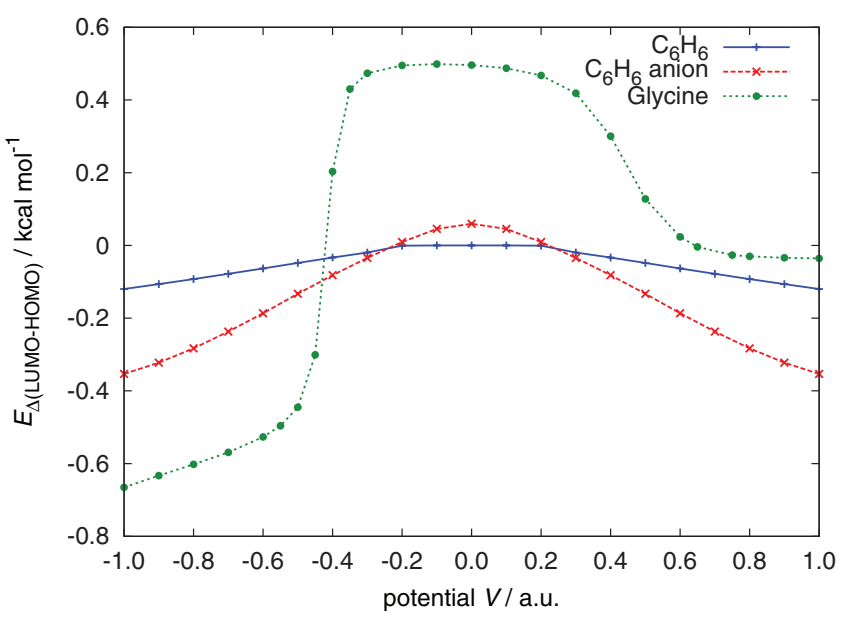

(b) Image charge contributions

FIG. 6. Static field and image charge contributions (see main text for definitions) to the HOMO-LUMO gap for neutral benzene (blue line), glycine (green line), and benzene anion (red line) with varying applied potential, $V$, an $x y$-plate area of $108 \times 108 \mathrm{bohr}^{2}$, and $\Delta z=54 \mathrm{bohr}$.

significant for glycine, not the benzene anion. Even though the anion orbitals are shifted more by the image potential, the effect is quite similar on both the HOMO and LUMO, so the gap changes little compared to glycine for most values of $V$. It is also interesting that the benzene curves have very similar shapes in both figures, regardless of the anion charge. In contrast, the glycine curve has a much more complex structure, reflecting the subtle changes in the electronic structure as both the static field and image charge potential change.

\section{CONCLUSION}

We have presented a new model and computer implementation for the treatment of molecular systems in arbitrary electrostatic environments. Our work is inspired by the continuum solvent models, but has a very different application focus in mind, for example, the simulation of molecules in nano-electronic devices. More precisely, our approach defines a system-environment model in which the effect of the environment is completely determined by the specification of a fixed electrostatic potential on the boundary interface, which 
is known a priori. In this way, the environment field must be solved self-consistently with the molecular system.

We have systematically explored the behaviour of our model in a number of controlled tests, making a detailed comparison with the simpler picture of a static external field, which is not solved self-consistently. The difference, which we term "the image charge effect," is found to strongly depend on the multipole character of the system's total charge distribution. Thus, it is quite small in the charge-neutral, quadrupolar, and benzene system, but somewhat larger in the dipolar (zwitterionic) glycine case. Similarly, it is quite significant in the charged benzene anion, where it modifies the total molecular energy by between 1 and $20 \mathrm{kcal} / \mathrm{mol}$, which is comparable in size to the changes induced by a static field of strength $1 \mathrm{~V}^{-1}$. As a result, we found that the benzene anion can actually be stabilized relative to neutral benzene when confined inside a grounded cavity.

In addition to total energies, we also explored the effect on orbital energy levels and the HOMO-LUMO gap, which reflects more subtle changes to the molecular orbitals than a constant energy shift. In this case, the image charge effect was similar in magnitude for all the molecules, modifying the gap by approximately $0.1-0.5 \mathrm{kcal} / \mathrm{mol}$, even at zero applied field. Nevertheless, this change is somewhat overwhelmed by the effect of a non-zero static bias. For example, a field strength of about $1 \mathrm{~V}^{-1}$ adjusts the gap by two orders of magnitude more than the image charge effect.

We conclude that image charge effects are often subtle, but in special cases (such as charged systems) or in very accurate work, a careful inclusion of their contributions can be important. Since electron transport properties are known to be rather sensitive to changes in molecular orbitals and their energy levels, it would be interesting to test the CheESE model in such cases as a future study.

\section{ACKNOWLEDGMENTS}

This work was supported by the National Science Foundation (NSF) under Contract No. NSF-PHY-0835713, the Defense Advanced Research Project Agency (DARPA) under Contract No. FA9550-08-1-0285, and the Defense Threat Reduction Agency under Contract No. HDTRA1-10-1-0046. This work was partially conducted while R.O.A. was a Giorgio Ruffolo Fellow in the Sustainability Science Program at Harvard University's Center for International Development. Computations in this paper were run on the Odyssey cluster supported by the FAS Research Computing Group. The authors would like to thank Dr. Michael Stopa, Dr. Xavier Andrade, and David Tempel for stimulating discussions in the early stages of this work.

\section{APPENDIX: A VARIATIONAL SCHEME FOR ELECTROSTATIC ENVIRONMENT EFFECTS}

In Sec. II D, we described an iterative scheme for obtaining the CheESE ground state from the time-independent Schrödinger equation, where $\Phi_{\text {env }}(\mathbf{r})$ is fixed at each iteration and then updated according to the new ground state. In the DFT case, the derivation of the KS equations for the calcu- lation at each iteration is therefore analogous to the conventional one. In particular, the functional derivative of $E_{\mathrm{tot}}^{\mathrm{sys}}$ in Eq. (28) with respect to the system density recovers the KS operator in the usual variational way, since $\Phi_{\text {env }}(\mathbf{r})$ is densityindependent at each iteration.

On the other hand, it is also possible to consider a noniterative algorithm, in which $\Phi_{\text {env }}(\mathbf{r})$ is not held fixed, and therefore explicitly depends on $\rho_{\text {sys }}(\mathbf{r})$. In this case, we cannot obtain the ground state density by simply minimizing the energy in Eq. (28); for example, in the DFT case, the functional derivative with respect to the system density would now introduce a spurious factor of two in the KS operator (see below). Instead, following Sanhueza et al., ${ }^{61}$ the ground state solution of the time-independent Schrödinger equation with a nonlinear Hamiltonian, $\hat{H}_{\mathrm{e}}^{\text {eff }}$, may be found by minimizing an alternative energy-like functional,

$$
J=\left\langle\tilde{\Psi}\left|\hat{H}_{\mathrm{e}}^{\mathrm{eff}}+\hat{G}\right| \tilde{\Psi}\right\rangle
$$

with respect to a complete set of trial wavefunctions, $\tilde{\Psi}$, where $\hat{G}$ depends on the form of the nonlinearity.

To obtain the form of $\hat{G}$ in the case of the CheESE Hamiltonian, we may first recognize, using a Green's function formalism, that $\Phi_{\text {image }}(\mathbf{r})$ depends linearly on the system density,

$$
\Phi_{\text {image }}(\mathbf{r})=-\int \rho_{\text {sys }}\left(\mathbf{r}^{\prime}\right) F\left(\mathbf{r}, \mathbf{r}^{\prime}\right) \mathrm{d} \mathbf{r}^{\prime},
$$

where $F(\mathbf{r}, \mathbf{r} /)$ depends only on the geometry of the system boundary, $\Omega_{\text {sys }}$; the precise form of $F\left(\mathbf{r}, \mathbf{r}^{\prime}\right)$ does not concern us here. More specifically, we can decompose $\Phi_{\text {image }}(\mathbf{r})$ into electronic and nuclear contributions,

$$
\Phi_{\text {image }}^{\text {elec }}(\mathbf{r})=\int \rho_{\text {sys }}^{\text {elec }}\left(\mathbf{r}^{\prime}\right) F\left(\mathbf{r}, \mathbf{r}^{\prime}\right) \mathrm{d} \mathbf{r}^{\prime},
$$

$$
\Phi_{\text {image }}^{\text {nuc }}(\mathbf{r})=-\int \rho_{\text {sys }}^{\text {nuc }}\left(\mathbf{r}^{\prime}\right) F\left(\mathbf{r}, \mathbf{r}^{\prime}\right) \mathrm{d} \mathbf{r}^{\prime} .
$$

According to Sanhueza et al. ${ }^{61}$ nonlinearities of this form are associated with an operator $\hat{G}$, given by

$$
\hat{G}=\frac{1}{2} \sum_{i}^{N} \Phi_{\text {image }}^{\text {elec }}\left(\mathbf{r}_{i}\right) .
$$

Note that we are not concerned with minimizing over the nuclear degrees of freedom.

In the DFT case, the electronic energy is given by

$$
E_{\text {elec }}^{\text {sys }}=E_{0}^{\mathrm{DFT}}-\int \rho_{\mathrm{sys}}^{\text {elec }}(\mathbf{r}) \Phi_{\mathrm{env}}(\mathbf{r}) \mathrm{d} \mathbf{r},
$$

but using the above approach, we can see, in comparison, that $J$ is given by

$$
J=E_{0}^{\mathrm{DFT}}-\int \rho_{\text {sys }}^{\text {elec }}(\mathbf{r})\left[\Phi_{\text {static }}+\Phi_{\text {image }}^{\text {nuc }}+\frac{1}{2} \Phi_{\text {image }}^{\text {elec }}\right] \mathrm{d} \mathbf{r} .
$$

Now, the functional derivative of $J$ with respect to $\rho_{\text {sys }}^{\text {elec }}(\mathbf{r})$, using Eq. (A3), returns the expected KS operator given in Eq. (27). However, the minimum $J$ does not equal the CheESE ground state electronic energy. Instead, if the density which 
minimizes $J$ is denoted $\rho_{\text {sys }}^{*}(\mathbf{r})$, then $E_{\text {elec }}^{\text {sys }}\left[\rho_{\text {sys }}^{*}\right]$ is the correct ground state energy, in the sense of being an (approximate) eigenvalue of the electronic Schrödinger equation (17). It should be stressed that this is the reason we quote all our results in terms of $E_{\mathrm{tot}}^{\mathrm{sys}}$, even though it is not a minimum (in general) for the ground state density!

${ }^{1}$ L. Vogt, R. Olivares-Amaya, S. Kermes, Y. Shao, C. Amador-Bedolla, and A. Aspuru-Guzik, J. Phys. Chem. A 112, 2049 (2008).

${ }^{2}$ I. S. Ufimtsev and T. J. Martinez, Comput. Sci. Eng. 10, 26 (2008).

${ }^{3}$ I. S. Ufimtsev and T. J. Martinez, J. Chem. Theory Comput. 5, 1004 (2009).

${ }^{4}$ R. Olivares-Amaya, M. A. Watson, R. G. Edgar, L. Vogt, Y. Shao, and A. Aspuru-Guzik, J. Chem. Theory Comput. 6, 135 (2010).

${ }^{5}$ M. Watson, R. Olivares-Amaya, R. G. Edgar, and A. Aspuru-Guzik, Comput. Sci. Eng. 12, 40 (2010).

${ }^{6}$ G. C. Schatz, Proc. Natl. Acad. Sci. U.S.A. 104, 6885 (2007).

${ }^{7}$ Advances in the Atomic-Scale Modeling of Nanosystems and Nanostructured Materials, Lecture Notes in Physics Vol. 795 , edited by C. Massobrio, H. Bulou, and C. Goyhenex (Springer, Heidelberg, 2010).

${ }^{8}$ Nanomaterials: Design and Simulation, Theoretical and Computational Chemistry Vol. 18, edited by P. B. Balbuena and J. M. Seminario (Elsevier, Amsterdam, 2007).

${ }^{9}$ Surface-Enhanced Raman Scattering: Physics and Applications, Topics in Applied Physics No. 103, K. Kneipp, M. Moskovits, and H. Kneipp (Springer, Berlin, 2006).

${ }^{10}$ R. Aroca, Surface Enhanced Vibrational Spectroscopy (Wiley, Chichester, 2006).

${ }^{11}$ E. C. Le Ru and P. G. Etchegoin, Principles of Surface-Enhanced Raman Spectroscopy and Related Plasmonic Effects (Elsevier, Amsterdam, 2008).

${ }^{12}$ P. L. Stiles, J. A. Dieringer, N. C. Shah, and R. P. Van Duyne, Annu. Rev. Anal. Chem. 1, 601 (2008).

${ }^{13}$ A. Campion and P. Kambhampati, Chem. Soc. Rev. 27, 241 (1998).

${ }^{14}$ A. Pecchia and A. D. Carlo, Rep. Prog. Phys. 67, 1497 (2004).

${ }^{15}$ W. Y. Kim, Y. C. Choi, S. K. Min, Y. Cho, and K. S. Kim, Chem. Soc. Rev. 38, 2319 (2009).

${ }^{16}$ H. Phillips, A. Prociuk, and B. D. Dunietz, J. Chem. Phys. 134, 054708 (2011).

${ }^{17}$ S. Kuhn, G. Mori, M. Agio, and V. Sandoghdar, Mol. Phys. 106, 893 (2008).

${ }^{18}$ M. Tsutsui, M. Taniguchi, K. Yokota, and T. Kawai, Nat. Nanotechnol. 5, 286 (2010).

${ }^{19}$ P.-O. Löwdin, J. Chem. Phys. 19, 1396 (1951).

${ }^{20} \mathrm{R}$. McWeeny, in Advances in Quantum Chemistry, edited by J. R. Sabin, M. C. Zerner, E. Brändas, S. Wilson, J. Maruani, Y. G. Smeyers, P. J. Grout, and R. McWeeny (Academic, New York, 1998), Vol. 31, pp. 15-35.

${ }^{21}$ K. Kitaura, E. Ikeo, T. Asada, T. Nakano, and M. Uebayasi, Chem. Phys. Lett. 313, 701 (1999).

${ }^{22}$ M. S. Gordon, J. M. Mullin, S. R. Pruitt, L. B. Roskop, L. V. Slipchenko, and J. A. Boatz, J. Phys. Chem. B 113, 9646 (2009).

${ }^{23}$ Y. Okiyama, T. Nakano, K. Yamashita, Y. Mochizuki, N. Taguchi, and S. Tanaka, Chem. Phys. Lett. 490, 84 (2010).

${ }^{24}$ T. Fujita, T. Nakano, and S. Tanaka, Chem. Phys. Lett. 506, 112 (2011).

${ }^{25}$ J. Tomasi and M. Persico, Chem. Rev. 94, 2027 (1994).

${ }^{26}$ J. Tomasi, B. Mennucci, and R. Cammi, Chem. Rev. 105, 2999 (2005).

${ }^{27}$ A. Klamt and G. Schüürmann, J. Chem. Soc., Perkin Trans. 2 1993, 799.
${ }^{28}$ A. Klamt, J. Phys. Chem. 99, 2224 (1995).

${ }^{29}$ A. Warshel and M. Levitt, J. Mol. Biol. 103, 227 (1976).

${ }^{30}$ M. J. Field, P. A. Bash, and M. Karplus, J. Comput. Chem. 11, 700 (1990).

${ }^{31}$ H. M. Senn and W. Thiel, in Atomistic Approaches in Modern Biology, edited by M. Reiher (Springer, Berlin Heidelberg, 2007), Vol. 268, pp. 173290.

${ }^{32}$ G. C. Schatz, Acc. Chem. Res. 17, 370 (1984).

${ }^{33}$ M. Moskovits, Rev. Mod. Phys. 57, 783 (1985).

${ }^{34}$ M. Stockman, in Surface-Enhanced Raman Scattering, edited by K. Kneipp, M. Moskovits, and H. Kneipp (Springer, Berlin Heidelberg, 2006), Vol. 103, Chap. 3, pp. 47-65.

${ }^{35}$ A. Otto, J. Raman Spectrosc. 36, 497 (2005).

${ }^{36}$ L. Zhao, L. Jensen, and G. C. Schatz, J. Am. Chem. Soc. 128, 2911 (2006).

${ }^{37}$ S. M. Morton and L. Jensen, J. Am. Chem. Soc. 131, 4090 (2009).

${ }^{38}$ S. K. Saikin, R. Olivares-Amaya, D. Rappoport, M. Stopa, and A. AspuruGuzik, Phys. Chem. Chem. Phys. 11, 9401 (2009).

${ }^{39}$ K. Yee, IEEE Trans. Antennas Propag. 14, 302 (1966).

${ }^{40}$ A. Taflove and S. C. Hagness, Computational Electrodynamics: The FiniteDifference Time-Domain Method, 3rd ed. (Artech House, Norwood, MA, 2005).

${ }^{41}$ D. J. Masiello and G. C. Schatz, Phys. Rev. A 78, 042505 (2008).

${ }^{42}$ D. J. Masiello and G. C. Schatz, J. Chem. Phys. 132, 064102 (2010).

${ }^{43}$ J. M. McMahon, S. K. Gray, and G. C. Schatz, Phys. Rev. Lett. 103, 097403 (2009).

${ }^{44}$ J. M. McMahon, S. K. Gray, and G. C. Schatz, Nano Lett. 10, 3473 (2010).

${ }^{45}$ H. Chen, J. M. McMahon, M. A. Ratner, and G. C. Schatz, J. Phys. Chem. C 114, 14384 (2010).

${ }^{46}$ S. Corni and J. Tomasi, J. Chem. Phys. 116, 1156 (2002).

${ }^{47} \mathrm{~S}$. Corni and J. Tomasi, in Surface-Enhanced Raman Scattering, Topics in Applied Physics Vol. 103, edited by K. Kneipp, M. Moskovits, and H. Kneipp (Springer, Berlin, Heidelberg, 2006), pp. 105-123.

${ }^{48}$ R. Olivares-Amaya, M. Stopa, X. Andrade, M. A. Watson, and A. AspuruGuzik, J. Phys. Chem. Lett. 2, 682 (2011).

${ }^{49}$ A. Castro, H. Appel, M. Oliveira, C. A. Rozzi, X. Andrade, F. Lorenzen, M. A.L. Marques, E. K.U. Gross, and A. Rubio, Phys. Status Solidi B 243, 2465 (2006), see http://www.tddft.org/programs/octopus/.

${ }^{50}$ P. Pulay, Chem. Phys. Lett. 73, 393 (1980).

${ }^{51}$ M. A. Watson, Y. Kurashige, T. Nakajima, and K. Hirao, J. Chem. Phys. 128, 054105 (2008).

${ }^{52}$ L. E. McMurchie and E. R. Davidson, J. Comput. Phys. 26, 218 (1978).

${ }^{53}$ DALTON2011, a molecular electronic structure program (2011), see http://www.daltonprogram.org.

${ }^{54}$ A. D. Becke, J. Chem. Phys. 98, 5648 (1993).

${ }^{55}$ S. R. Kass, J. Am. Chem. Soc. 127, 13098 (2005).

${ }^{56}$ Y. C. Choi, W. Y. Kim, K.-S. Park, P. Tarakeshwar, K. S. Kim, T.-S. Kim, and J. Y. Lee, J. Chem. Phys. 122, 094706 (2005).

${ }^{57}$ W. Y. Kim and K. S. Kim, Acc. Chem. Res. 43, 111 (2010).

${ }^{58}$ M. J. W. Boness, I. W. Larkin, J. B. Hasted, and L. Moore, Chem. Phys. Lett. 1, 292 (1967).

${ }^{59}$ J. C. Rienstra-Kiracofe, C. J. Barden, S. T. Brown, and H. F. Schaefer III, J. Phys. Chem. A 105, 524 (2001).

${ }^{60}$ W. Y. Kim and K. S. Kim, Acc. Chem. Res. 43, 111 (2010).

${ }^{61}$ J. E. Sanhueza, O. Tapia, W. G. Laidlaw, and M. Trsic, J. Chem. Phys. 70, 3096 (1979).

${ }^{62}$ A. D. Miller, I. Bezel, K. J. Gaffney, S. Garrett-Roe, S. H. Liu, P. Szymanski, and C. B. Harris, Science 297, 1163 (2002). 